\title{
Seasonal changes in paralarval cephalopod communities on the southwest coast of Baja California Sur (spring and autumn 2003)
}

\section{Cambios estacionales en las comunidades de paralarvas de cefalópodos en la costa suroccidental de Baja California Sur (primavera-otoño de 2003)}

\author{
Rubén Melvyn García-Guillén, Roxana De Silva-Dávila*, Raymundo Avendaño-Ibarra \\ Instituto Politécnico Nacional, Centro Interdisciplinario de Ciencias Marinas, Departamento de Plancton y \\ Ecología Marina, Av. IPN s/n, colonia Playa Palo de Sta. Rita, CP 23096, La Paz, Baja California Sur, Mexico.
}

* Corresponding author. E-mail: rdesilva@ipn.mx

\begin{abstract}
We recorded seasonal changes in the paralarval cephalopod communities collected off the southwestern coast of Baja California Sur, Mexico, during 2003. Simultaneous surface and oblique trawls were carried out with CalCOFI-type nets and CTD casts during spring and autumn 2003 on board the R/V Río Suchiate (Secretaría de Marina), at 85 sampling stations (Magdalena Bay to Cape San Lucas). The paralarval cephalopod community predominantly showed tropical affinity in both seasons. During autumn, 11 tropical taxa, 2 cosmopolitan taxa, and one temperate taxon were incorporated to the paralarval community recorded previously in spring and were associated with the decrease in Subarctic Water and the entrance of warm water masses to the study region, modifying the community indexes between seasons. The canonical correspondence analysis showed 2 groups significantly correlated with chlorophyll $a$ concentrations and zooplankton volumes. Oblique tows provide better information on the paralarval cephalopod community, while surface tows can be an efficient method for monitoring and evaluating the hatching areas of the jumbo Pacific squid Dosidicus gigas through their paralarvae, which are included in the Sthenoteuthis oualaniensis-Dosidicus gigas complex (SD complex).
\end{abstract}

Key words: paralarvae, community structure, SD complex, Baja California Sur.

RESUMEN. Se registró el cambio estacional en las comunidades de paralarvas de cefalópodos recolectadas frente a la costa suroccidental de Baja California Sur, México, durante 2003. Para lo anterior, se realizaron arrastres superficiales y oblicuos simultáneos con redes tipo CalCOFI y lances de CTD en 85 estaciones de muestreo (de bahía Magdalena a cabo San Lucas) durante primavera y otoño de 2003 a bordo del B/I Río Suchiate (Secretaría de Marina). La comunidad de paralarvas de cefalópodos fue mayormente tropical en ambas temporadas. Durante el otoño se adicionaron 11 taxones tropicales, 2 cosmopolitas y 1 templado a la comunidad registrada previamente en primavera, todos relacionados con la disminución de Agua Subártica y el ingreso de masas de agua cálida a la región; esto modificó los índices comunitarios entre las temporadas. El análisis de correspondencia canónica mostró la formación de 2 grupos correlacionados significativamente con la concentración de clorofila $a$ y el volumen de zooplancton. Los arrastres oblicuos dan mayor información sobre la comunidad de paralarvas, mientras que los superficiales resultan eficientes para el seguimiento y la evaluación de las áreas de eclosión del calamar gigante del Pacífico, Dosidicus gigas, a través de sus paralarvas incluidas en el complejo Sthenoteuthis oualaniensis-Dosidicus gigas (complejo SD).

Palabras clave: paralarvas, estructura de la comunidad, complejo SD, Baja California Sur.

\section{INTRODUCTION}

Most cephalopods have rapid growth rates, $r$-selection strategy, and short life cycles (Boyle and Rodhouse 2005). Their role as keystone species in marine food webs (Piatkowski et al. 2001) and their sensitivity to physicochemical variations in the water column, variability in ocean circulation at different scales, and food availability make them potential indicators of environmental change (Moreno et al. 2009, Camarillo-Coop et al. 2011, Zaragoza et al. 2014). The stage in the cephalopod life cycle that is most sensitive to environmental variations is the paralarval phase. Paralarvae occur at the early post-hatching stage in most cephalopods (Young and Harman 1988); they are planktonic and thus depend on the effects of ocean circulation (Boyle and Rodhouse 2005).

\section{INTRODUCCIÓN}

En su mayoría los cefalópodos presentan un rápido crecimiento, estrategia $\mathrm{r}$ y un ciclo de vida corto (Boyle y Rodhouse 2005). Su posición como componentes clave en las tramas tróficas marinas (Piatkowski et al. 2001) y su sensibilidad a las variaciones fisicoquímicas en la columna de agua, a la variabilidad de diferente escala en la circulación oceánica y a la disponibilidad de alimento, los convierten en indicadores potenciales del cambio ambiental (Moreno et al. 2009, Camarillo-Coop et al. 2011, Zaragoza et al. 2014). La etapa más sensible a la variación ambiental en el ciclo de vida de los cefalópodos es la de paralarvas. Las paralarvas representan el estadio inicial posteclosión en la mayoría de los cefalópodos (Young y Harman 1988); son planctónicas y, por 
In Mexico, mainly off northwestern Mexico and off the Gulf of Tehuantepec, the effects of the regional oceanography and of some physical, chemical, and biological variables on paralarvae distribution have been analyzed at different temporal and spatial scales. The paralarval community structure from Ensenada, Baja California, to Point Abreojos, Baja California Sur, during the 1997-2001 El Niño/Southern Oscillation (ENSO) event was modulated by physical variables associated with the latitudinal extension and retrieval of water masses, which prompted a significant change in community indexes and in the dominance of species whose biogeographic affinities were associated with anomalous warming or cooling events (Durazo and Baumgartner 2002, Granados-Amores et al. 2010). During 2004-2007, when ENSO influence was not significant in the Gulf of California, 3 paralarval assemblages were recurrently found in surface water masses, indicating that tropical species were transported into the gulf and enriched the resident community (De Silva-Dávila et al. 2015). On the other hand, size distribution and oceanic and coastal paralarval communities in the Gulf of Tehuantepec were influenced by 2 eddies, an upwelling front, and the hydrodynamics over the continental shelf (Aceves-Medina et al. 2017). The southwestern coast of Baja California Sur is one of the least studied regions in northwestern Mexico; thus, the seasonal effect of ocean dynamics on paralarval communities there is mostly unknown.

The Sthenoteuthis oualaniensis-Dosidicus gigas complex (SD complex) is a frequent and abundant component of paralarval communities off the Pacific coasts of Mexico. This species complex is formed by paralarvae, measuring $\leq 3.0-4.0 \mathrm{~mm}$ mantle length (ML), that cannot be morphologically differentiated to species (Staaf et al. 2011, De Silva-Dávila 2013). This complex has been positively correlated with high temperatures at the 3 above mentioned regions, despite the different environments, and paralarvae measuring $\leq 2.0 \mathrm{~mm}$ ML evidenced the occurrence of spawning events in all these regions (Granados-Amores et al. 2010, De Silva-Dávila 2013, Aceves-Medina et al. 2017), exhibiting the reproductive plasticity and opportunistic nature of these species (Hoving et al. 2013, Hernández-Muñoz et al. 2016).

Findings on paralarval abundance are based on studies using only one sampling gear. However, the use of different net towing methods can affect the structure of collected zooplankton communities, and cephalopod communities are not an exception (Saito 1994, Staaf et al. 2013). The use of different complementary sampling gears can provide a stronger database for the analysis of cephalopod paralarval communities (De Silva-Dávila et al. 2015).

In spring the California Current appears as an intense equatorward flow that runs parallel to the coast from Ensenada to Point Abreojos. In autumn this flow weakens and tends to form sinuous meanders (Durazo 2015). The oceanic region off the southwestern coast of Baja California Sur lo tanto, altamente dependientes del efecto de la circulación oceánica (Boyle y Rodhouse 2005).

En México, el efecto de la oceanografía regional y de algunas variables físicas, químicas y biológicas en la distribución de las paralarvas, a diferentes escalas de tiempo y espacio, ha sido analizado, principalmente frente a la zona noroeste y frente al golfo de Tehuantepec. Desde Ensenada, Baja California, hasta punta Abreojos, Baja California Sur, la estructura de la comunidad de paralarvas durante El Niño/ Oscilación del Sur (ENOS) en 1997-2001 estuvo modulada por las variables físicas asociadas al avance y retroceso latitudinal de las masas de agua, lo que promovió un cambio significativo en los índices comunitarios y en la dominancia de especies cuyas afinidades biogeográficas se relacionaron con el calentamiento o el enfriamiento anómalos registrados (Durazo y Baumgartner 2002, Granados-Amores et al. 2010). En el golfo de California, durante 2004-2007, sin influencia significativa de ENOS, 3 asociaciones recurrentes de paralarvas se acoplaron a las masas de agua superficiales, lo que evidencia el transporte de especies tropicales al interior del golfo y el enriquecimiento de la comunidad residente (De Silva-Dávila et al. 2015). Por otro lado, en el golfo de Tehuantepec, la distribución de tallas y las comunidades oceánica y costera de paralarvas estuvieron influenciadas por 2 remolinos y un frente de surgencia, así como por la hidrodinámica sobre la plataforma continental (Aceves-Medina et al. 2017). La costa suroccidental de Baja California Sur es una de las regiones menos muestreadas de la zona noroeste, por lo que se desconoce, en su mayoría, el efecto estacional de la dinámica oceanográfica en las comunidades de paralarvas.

Un componente abundante y frecuente de las comunidades de paralarvas en las aguas del Pacífico frente a las costas de México es el complejo Sthenoteuthis oualaniensis-Dosidicus gigas (complejo SD). Este complejo está formado por paralarvas de estas especies que, a tallas $\leq 3.0-4.0 \mathrm{~mm}$ de longitud de manto (LM), no se pueden diferenciar morfológicamente a nivel de especie (Staaf et al. 2011, De Silva-Dávila 2013). Este complejo se ha correlacionado positivamente con temperaturas altas en las 3 regiones mencionadas, a pesar de los diferentes ambientes de distribución, y sus paralarvas de tallas $\leq 2.0 \mathrm{~mm}$ LM evidenciaron eventos de desove en todas ellas (Granados-Amores et al. 2010, De Silva-Dávila 2013, Aceves-Medina et al. 2017), lo cual refleja la plasticidad reproductiva de estas especies y su naturaleza oportunista (Hoving et al. 2013, Hernández-Muñoz et al. 2016).

Por otra parte, la mayoría de los antecedentes de la abundancia de paralarvas están basados en estudios realizados con un solo arte de muestreo. Sin embargo, el uso de diferentes tipos de arrastre influye en la estructura de las comunidades del zooplancton recolectadas, y las de cefalópodos no son la excepción (Saito 1994, Staaf et al. 2013). El uso de diferentes artes de muestreo en forma complementaria resulta en una mejor base de datos para el análisis de las comunidades de paralarvas de cefalópodos (De Silva-Dávila et al. 2015). 
(SWC) is the southernmost portion of the California Current System, where temperate water of subarctic origin and warm water of subtropical origin seasonally converge (Durazo and Baumgartner 2002). In April the equatorward flow is constant, with a meander flowing offshore south of Magdalena Bay; in October, the effect of the California Current decreases and the dominant flow has a poleward direction, passing near the coast up to the Gulf of Ulloa (Zaitsev et al. 2014).

The objective of this study was to determine the seasonal differences between the communities of cephalopod paralarvae collected with 2 types of net tows off the SWC and to relate them to changes in oceanographic conditions during spring and autumn 2003. The information produced here, especially information on paralarvae of the SD complex, which includes the fishery resource $D$. gigas, will improve knowledge for better assessment of this resource on both sides of the Baja California Peninsula.

\section{MATERIALS AND METHODS}

Eighty-five zooplankton samples were collected during 2 oceanographic campaigns aboard the R/V Río Suchiate of the Secretaría de Marina. These campaigns were carried out off the SWC, from Magdalena Bay to Cape San Lucas (Fig. 1a), between 30 April and 14 May (spring) (Fig. 1b) and between 30 September and 11 October (autumn) 2003 (Fig. 1c).

At each sampling station, CTD casts (SBE 19 SeaCAT, maximum depth $500 \mathrm{~m}$ ) were performed to determine the environment in the water column (Durazo and Baumgartner 2002, Avendaño-Ibarra et al. 2010). Zooplankton was collected by making simultaneous surface and oblique tows with CalCOFI nets $(60-\mathrm{cm}$ diameter and $505-\mu \mathrm{m}$ mesh) equipped with calibrated flowmeters, following Kramer et al. (1972). Maximum depth was $1 \mathrm{~m}$ for surface tows and $223 \mathrm{~m}$ for oblique tows. Samples were fixed in buffered $4 \%$ formaldehyde (Kramer et al. 1972) and displacement volumes were determined (Beers 1976). Cephalopod paralarvae were separated from the unfractionated samples and then identified and measured (Roper and Voss 1983, Sweeney et al. 1992, De Silva-Dávila 2013) using a Stemi SV-11 stereoscope equipped with a calibrated ocular micrometer. Abundance was standardized to number of paralarvae per $1,000 \mathrm{~m}^{3}$ of filtered water $\left(\mathrm{PL} \cdot 1,000 \mathrm{~m}^{-3}\right)$ (Fleminger 1964).

A nonparametric analysis (Mann-Whitney $U$ test, $P<0.05$ ) was used to record significant differences between abundances, and traditional ecological indexes (richness, diversity, and dominance) (Margalef 1982) were used to analyze community structure between seasons and types of tow. The biogeographic affinity (temperate, tropical-subtropical, and cosmopolite) of paralarvae was determined based on the biogeographic affinity of adults (Jereb and Roper 2010, Jereb et al. 2014).

Taxon abundance distribution was determined with a two-way cluster analysis for each type of tow using
En términos ambientales, durante la primavera, en el área oceánica desde Ensenada hasta punta Abreojos, la corriente de California se presenta como un flujo intenso paralelo a la costa con dirección al ecuador. En otoño este flujo se debilita y tiende a la formación de meandros sinuosos (Durazo 2015). La región oceánica frente a la costa suroccidental de Baja California Sur (CSO) (Fig. 1a) es la porción más sureña del Sistema de la Corriente de California, donde estacionalmente convergen agua templada de origen subártico y agua cálida de origen subtropical (Durazo y Baumgartner 2002). En abril, el flujo hacia el ecuador es constante, con un meandro con dirección hacia fuera de la costa al sur de bahía Magdalena; en octubre, el efecto de la corriente de California disminuye y el flujo dominante se dirige hacia el polo, pasando cerca de la costa hasta el golfo de Ulloa (Zaitsev et al. 2014).

El objetivo de este estudio fue establecer las diferencias estacionales entre las comunidades de paralarvas de cefalópodos recolectadas con 2 tipos de arrastre frente a la $\mathrm{CSO}$, y relacionarlas con el cambio en las condiciones oceanográficas de primavera y otoño de 2003. La información, especialmente aquella sobre las paralarvas del complejo SD que incluye a las paralarvas del recurso pesquero D. gigas, ampliará el conocimiento para una mejor evaluación del recurso a ambos lados de la península de Baja California.

\section{MATERIALES Y MÉTODOS}

Se recolectaron 85 muestras de zooplancton en 2 campañas oceanográficas a bordo del B/I Río Suchiate de la Secretaría de Marina, Armada de México. Los cruceros se llevaron a cabo frente a la CSO, desde bahía Magdalena hasta cabo San Lucas (Fig. 1a), del 30 de abril al 14 de mayo (primavera) (Fig. 1b) y del 30 de septiembre al 11 de octubre (otoño) de 2003 (Fig. 1c).

En cada estación de muestreo se realizaron lances de CTD (modelo SBE 19 SeaCAT) (profundidad máxima de $500 \mathrm{~m}$ ) para determinar el ambiente en la columna de agua (Durazo y Baumgartner 2002, Avendaño-Ibarra et al. 2010). El zooplancton se recolectó mediante arrastres superficiales y oblicuos simultáneos usando redes CalCOFI $(60 \mathrm{~cm}$ de diámetro y $505 \mu \mathrm{m}$ de apertura de malla) provistas con flujómetros calibrados, de acuerdo con los criterios de Kramer et al. (1972). Los arrastres superficiales se realizaron a una profundidad máxima de $1 \mathrm{~m}$ y los oblicuos hasta $223 \mathrm{~m}$. Las muestras se fijaron en formaldehido al $4 \%$ neutralizado (Kramer et al. 1972), y se determinó su volumen desplazado (Beers 1976). Las paralarvas de cefalópodos se separaron de las muestras sin fraccionar, y se identificaron y midieron (Roper y Voss 1983, Sweeney et al. 1992, De Silva-Dávila 2013) utilizando un estereoscopio Stemi SV-11 provisto de un micrómetro ocular calibrado. La abundancia se estandarizó a número de paralarvas por $1,000 \mathrm{~m}^{3}$ de agua filtrada $\left(\mathrm{PL} \cdot 1,000 \mathrm{~m}^{-3}\right)$ (Fleminger 1964).

Se registraron diferencias significativas entre la abundancia utilizando un análisis no paramétrico ( $U$ de Mann-Whitney, 
the Bray-Curtis dissimilarity index $(\beta=-0.25)$ and the PC-ORD 6.0 software package (McCune and Mefford 2011). Two primary abundance matrices per taxon (surface tow and oblique tow) with $\log 10(x+1)$ transformed data were used, and 2 secondary matrices were made with the following qualitative variables: season (spring/autumn) and identified water mass at each sampling station. The speciesenvironment relationship was explored by performing a canonical correspondence analysis per type of tow with CANOCO 4.5, and a Monte Carlo test (999 permutations) (Ter Braak 1994). Two primary abundance matrices and 2 secondary environment matrices (surface tow and oblique tow) were used, excluding species that were present at only one sampling station. The environment matrix for surface tows included the variables temperature, salinity, and zooplankton volume at $1 \mathrm{~m}$ depth; and the environment matrix for oblique tows included temperature at $10 \mathrm{~m}$ depth, salinity at $10 \mathrm{~m}$ depth, and zooplankton volume in the oblique tows. Chlorophyll $a$ concentrations $\left(\mathrm{mg} \cdot \mathrm{m}^{-3}\right)$ obtained from
$P<0.05$ ), y los índices ecológicos tradicionales (riqueza, diversidad y dominancia) (Margalef 1982) se utilizaron para el análisis de la estructura de las comunidades entre temporadas y tipos de arrastre. Se determinó la afinidad biogeográfica de las paralarvas (templada, tropical-subtropical y cosmopolita) con base en la de los adultos (Jereb y Roper 2010, Jereb et al. 2014).

Se determinó la distribución de la abundancia de los taxones para cada tipo de arrastre mediante un análisis de agrupamiento (cluster) de 2 vías utilizando el índice de distancia de Bray-Curtis $(\beta=-0.25)$ y el programa PC-ORD 6.0 (McCune y Mefford 2011). Se utilizaron 2 matrices primarias de abundancia por taxón (superficial y oblicua) transformadas a $\log _{10}(x+1)$, y 2 matrices secundarias con las variables cualitativas temporada (primavera/otoño) y masa de agua identificada en cada estación de muestreo. Se exploró la relación especies-ambiente con un análisis de correspondencia canónica por tipo de arrastre mediante el programa CANOCO 4.5, y con una prueba de Montecarlo a
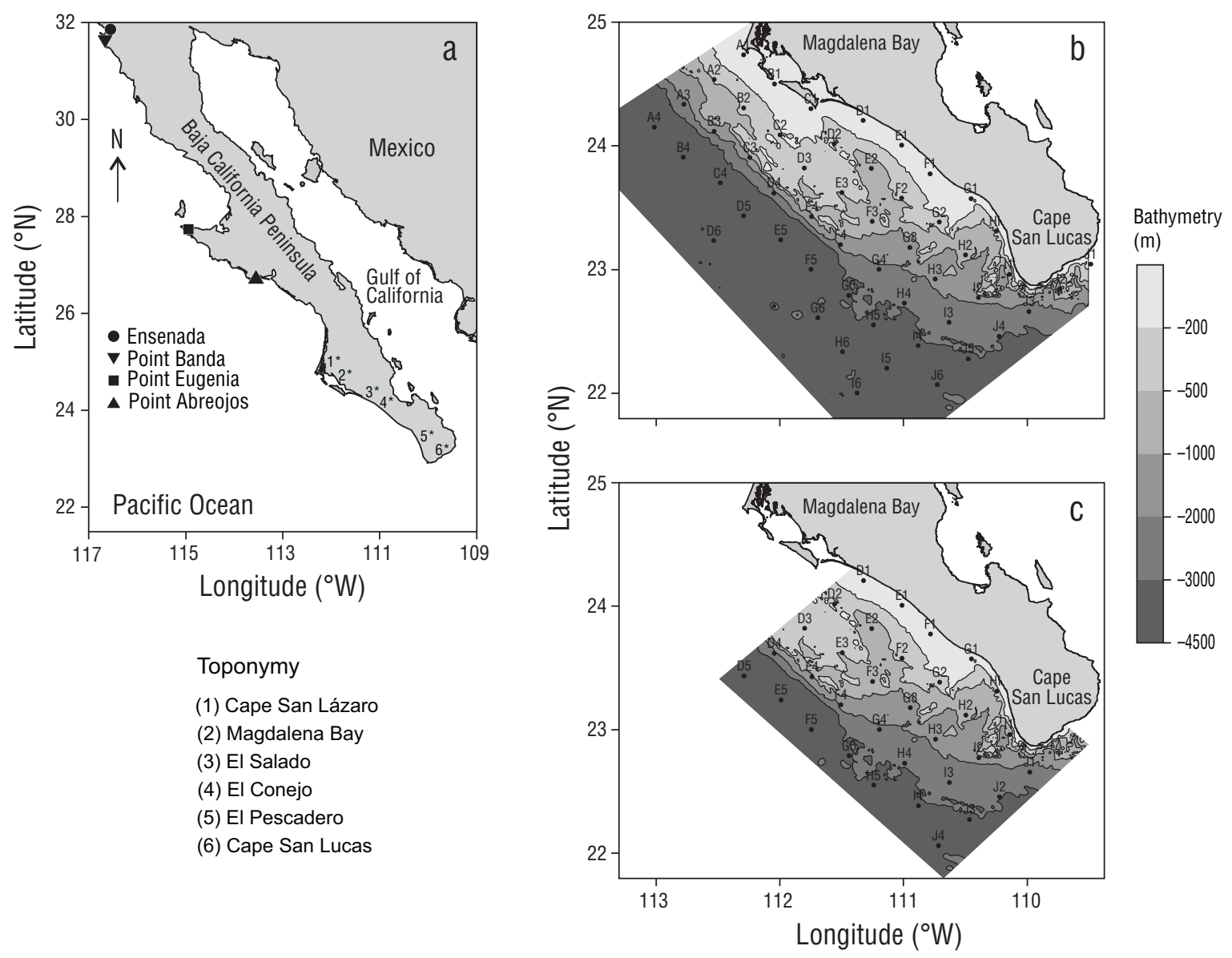

Figure 1. Study area off the southwestern coast of the Baja California Peninsula, Mexico (a), and sampling stations during spring (b) and autumn (c) 2003.

Figura 1. Área de estudio en la costa suroccidental de la península de Baja California, México (a), y estaciones de muestreo durante la primavera (b) y el otoño (c) de 2003. 
biweekly satellite images (resolution: $4 \mathrm{~km}^{2}$ ) during each sampling season (http://coastwatch.pfeg.noaa.gov/erddap/ index.html) were used for both matrices.

\section{ReSUlts}

\section{Environmental variables}

In spring conditions were cold (average $=18.5^{\circ} \mathrm{C}$ ), with a coast-ocean surface temperature gradient (minimum $=$ $13.5^{\circ} \mathrm{C}$ ) from Magdalena Bay to the north of El Pescadero, and maximum temperature $\left(22.5^{\circ} \mathrm{C}\right)$ was recorded to the south off Cape San Lucas (Fig. 2a). Transitional Water and Subarctic Water were recorded near the coast and off the coast, respectively, and a Subtropical Surface Water intrusion was recorded in the southern area (Fig. 2b). Chlorophyll $a$ concentrations showed an inverse gradient, with highest values $\left(3.2 \mathrm{mg} \cdot \mathrm{m}^{-3}\right)$ in the coastal zone off Magdalena Bay and El Salado and lowest values $\left(0.01 \mathrm{mg} \cdot \mathrm{m}^{-3}\right)$ in the oceanic zone (Fig. 2c). Zooplankton volumes at the surface were highest $\left(1,500 \mathrm{~mL} \cdot 1,000 \mathrm{~m}^{-3}\right)$ to the north of Magdalena Bay and to the south, off El Conejo (Fig. 2d). By contrast, conditions in autumn were warm. Sea surface temperature reached $29.2^{\circ} \mathrm{C}$ near the coast, with an average that was $9{ }^{\circ} \mathrm{C}$ higher than the average for spring (Fig. 2e). In autumn 2 Subtropical Surface Water intrusions in the oceanic zone, Transitional Water in the coastal and central oceanic zones, and a small Tropical Surface Water intrusion in the oceanic zone to the south were identified (Fig. 2f). Chlorophyll $a$ concentrations were low throughout the study area (average $=0.11 \mathrm{mg} \cdot \mathrm{m}^{-3}$ ) (Fig. 2g), and surface zooplankton volumes were lower (maximum $=600 \mathrm{~mL} \cdot 1,000 \mathrm{~m}^{-3}$ ) than the volumes observed for spring (Fig. 2h).

\section{Community structure}

A total of 257 cephalopod paralarvae were collected, and the corresponding standardized abundance was 2,591 PL $\cdot 1,000 \mathrm{~m}^{-3}$. Abundance of paralarvae in spring surface tows was higher than in oblique tows and higher than in both types of tows in autumn $(P<0.00024)$. In autumn more paralarvae were also collected at the surface, but abundances were not significantly different from abundances in oblique tows during that season (Table 1). Species richness and diversity were higher in oblique tows (both seasons), and the high dominance levels in spring surface tows was associated with the high abundance of SD complex paralarvae $\left(1,456 \mathrm{PL} \cdot 1,000 \mathrm{~m}^{-3}\right)$ and the lowest species richness.

The main differences in community composition between seasons were the presence of the Cranchiidae and Gonatidae families only during spring and the increase in species richness and morphotypes including the SD complex from 11 in spring to 19 in autumn (Table 2). Regarding biogeographic affinity, tropical and subtropical taxa dominated in both seasons and types of tows. Nevertheless, in autumn 11 tropical
999 permutaciones (Ter Braak 1994). Se utilizaron 2 matrices primarias de abundancia y 2 matrices secundarias ambientales (arrastre superficial y arrastre oblicuo) depurando las especies presentes solamente en una estación de muestreo. La matriz ambiental para los arrastres superficiales incluyó las variables temperatura, salinidad y volumen de zooplancton en el primer metro de profundidad, y la matriz ambiental para los arrastres oblicuos, las variables temperatura y salinidad a $10 \mathrm{~m}$ de profundidad y volumen de zooplancton del arrastre oblicuo. En ambas se utilizó la concentración de clorofila $a$ $\left(\mathrm{mg} \cdot \mathrm{m}^{-3}\right)$ obtenida de imágenes de satélite catorcenales en cada temporada de muestreo con una resolución de $4 \mathrm{~km}^{2}$ ( http://coastwatch.pfeg.noaa.gov/erddap/index.html).

\section{Resultados}

\section{Variables ambientales}

En primavera se registraron condiciones frías (promedio $=$ $18.5^{\circ} \mathrm{C}$ ) con un gradiente de temperatura superficial costaocéano (mínima $=13.5^{\circ} \mathrm{C}$ ) desde bahía Magdalena hasta el norte de El Pescadero, y la máxima temperatura $\left(22.5^{\circ} \mathrm{C}\right)$ se registró al sur frente a cabo San Lucas (Fig. 2a). También se registró Agua Transicional y Agua Subártica cerca y fuera de la costa, respectivamente, y una intrusión de Agua Superficial Subtropical en la zona sur (Fig. 2b). La concentración de clorofila $a$ presentó un gradiente inverso al de la temperatura, con los valores más altos $\left(3.2 \mathrm{mg} \cdot \mathrm{m}^{-3}\right)$ en la zona costera frente a bahía Magdalena y El Salado y los más bajos $\left(0.01 \mathrm{mg} \cdot \mathrm{m}^{-3}\right)$ en la zona oceánica (Fig. $\left.2 \mathrm{c}\right)$. Los volúmenes de zooplancton superficiales más altos $\left(1,500 \mathrm{~mL} \cdot 1,000 \mathrm{~m}^{-3}\right)$ se localizaron al norte de bahía Magdalena y al sur frente a El Conejo (Fig. 2d). En contraste, en otoño se registraron condiciones cálidas. La temperatura superficial del mar alcanzó los $29.2{ }^{\circ} \mathrm{C}$ cerca de la costa, con un promedio 9 grados más alto que en primavera (Fig. 2e). En esta temporada se identificaron 2 intrusiones de Agua Superficial Subtropical en la parte oceánica, Agua Transicional con mayor dominio en la zona costera y central oceánica y una pequeña intrusión de Agua Superficial Tropical al sur en la zona más oceánica (Fig. 2f). La concentración de clorofila $a$ fue baja en toda el área de estudio (promedio $=0.11 \mathrm{mg} \cdot \mathrm{m}^{-3}$ ) $($ Fig. $2 \mathrm{~g}$ ), y los volúmenes superficiales de zooplancton fueron inferiores (máximo $=600 \mathrm{~mL} \cdot 1,000 \mathrm{~m}^{-3}$ ) a los de primavera (Fig. 2h).

\section{Estructura de las comunidades}

Se recolectaron 257 paralarvas de cefalópodos, equivalentes a una abundancia estandarizada de 2,591 PL $\cdot 1,000 \mathrm{~m}^{-3}$. La abundancia en los arrastres superficiales de primavera fue mayor $(P<0.00024)$ que la abundancia en los arrastres oblicuos y que la abundancia en ambos tipos de arrastre de otoño. En otoño también se recolectaron más paralarvas en superficie, pero las abundancias no fueron significativamente diferentes de las abundancias en los muestreos oblicuos de 

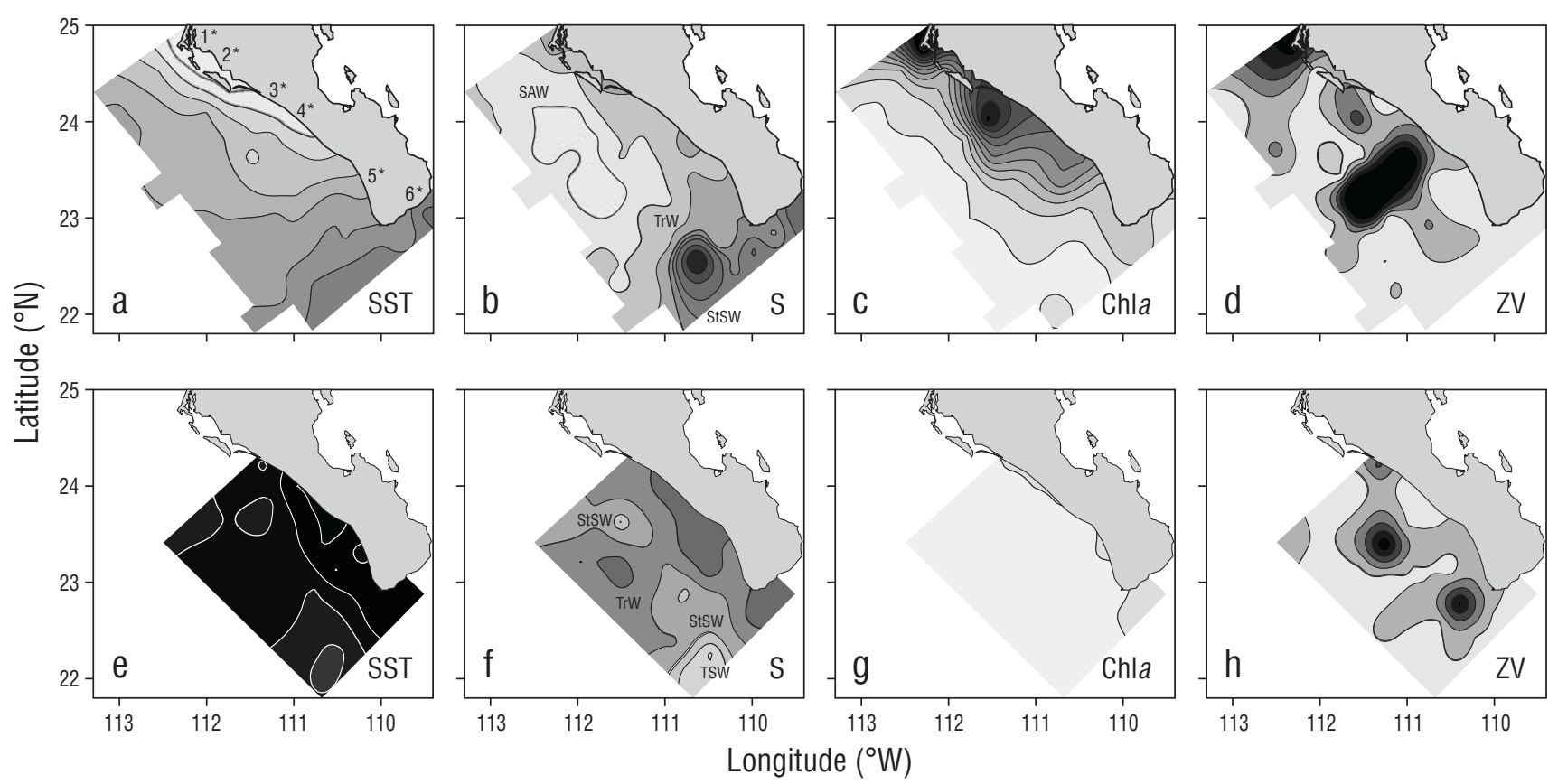

SST $\left({ }^{\circ} \mathrm{C}\right)$
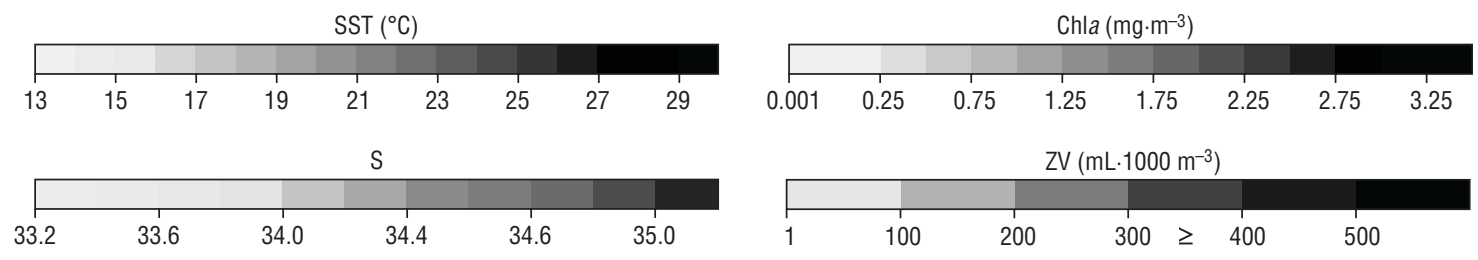

Figure 2. Distribution of environmental variables during spring $(\mathbf{a}, \mathbf{b}, \mathbf{c}, \mathbf{d})$ and autumn $(\mathbf{e}, \mathbf{f}, \mathbf{g}, \mathbf{h})$. Abbreviations: SST, sea surface temperature; S, salinity; Chla, chlorophyll a concentration; ZV, zooplankton volume; SAW, Subarctic Water; TrW, Transitional Water; StSW, Subtropical Surface Water; TSW, Tropical Surface Water. Locations: 1, Cape San Lázaro; 2, Magdalena Bay; 3, El Salado; 4, El Conejo; 5, El Pescadero; 6, Cape San Lucas.

Figura 2. Distribución de las variables ambientales durante la primavera (a, b, c, d) y el otoño (e, f, g, h). Abreviaturas: SST, temperatura superficial del mar; S, salinidad; Chla, concentración de clorofila a; ZV, volumen de zooplancton; SAW, Agua Subártica; TrW, Agua Transicional; StSW, Agua Superficial Subtropical; TSW, Agua Superficial Tropical. Localidades: 1, cabo San Lázaro; 2, bahía Magdalena; 3, El Salado; 4, El Conejo; 5, El Pescadero; 6, cabo San Lucas.

taxa, 2 cosmopolite taxa, and 1 temperate taxon were recorded in addition to those recorded in spring (Table 2).

Abundances for most species and morphotypes in both seasons were $<50 \mathrm{PL} \cdot 1,000 \mathrm{~m}^{-3}$, except for the $\mathrm{SD}$ complex and Abraliopsis sp. 1. In spring, the SD complex made up $95.1 \%$ and $37.4 \%$ of total abundances in surface and oblique tows, respectively; in autumn, it made up to $32.5 \%$ in surface tows and $39.1 \%$ in oblique tows. Abraliopsis sp. 1, recorded in autumn, made up to $30.9 \%$ of total abundance in surface tows and $14.4 \%$ in oblique tows.

\section{Statistical analysis}

The cluster analysis for surface tows showed the presence of 2 species-stations groups only during autumn in Transitional Water and Subtropical Surface Water, one characterized by the abundance and high frequency of Abraliopsis sp. 1 and the other by the low frequency of esa temporada (Tabla 1). La riqueza y la diversidad fueron mayores en los arrastres oblicuos (ambas temporadas), y la alta dominancia en los arrastres superficiales de primavera se relacionó con la alta abundancia del complejo SD $\left(1,456 \mathrm{PL} \cdot 1,000 \mathrm{~m}^{-3}\right)$ y con la más baja riqueza de especies.

Las principales diferencias en la composición de las comunidades entre temporadas fueron la presencia de las familias Cranchiidae y Gonatidae solo en primavera y el incremento en la riqueza de especies y morfotipos, incluso el complejo $\mathrm{SD}$, de 11 en primavera a 19 en otoño (Tabla 2). En cuanto a la afinidad biogeográfica, predominaron taxones tropicales y subtropicales en ambas temporadas y tipos de arrastres. Sin embargo, en otoño se registraron 11 taxones tropicales, 2 taxones cosmopolitas y 1 taxón templado adicionales a los registrados en primavera (Tabla 2 ).

La abundancia de la mayoría de las especies y morfotipos en ambas temporadas fue $<50 \mathrm{PL} \cdot 1,000 \mathrm{~m}^{-3}$, excepto para el complejo SD y Abraliopsis sp. 1. En primavera el complejo 
Argonauta sp. 3 and Japetella diaphana. A third group characterized by the SD complex was present during both seasons in all the identified water masses (Fig. 3a). Clustering for oblique tows showed a similar pattern. The first group, which included mainly stations sampled in autumn, was characterized by the presence of the SD complex in warm water masses and at 3 stations with Subarctic Water. The pelagic octopus $J$. diaphana and the jumbo squid $D$. gigas made up the second group, which was present in Transitional Water and Subarctic Water, and the third and fourth groups included species with low-frequency appearances in both seasons (Fig. 3b).

The canonical correspondence analysis for surface tows explained $92.6 \%$ of the cumulative variance in the speciesenvironment relation on the first 2 axes. Chlorophyll $a$ concentration (0.624) and zooplankton volume (0.899) on axes 1 and 2, respectively, showed the highest correlation. Similarly, for oblique tows the cumulative variance explained $71.4 \%$ of the species-environment relation, with zooplankton volume at $10 \mathrm{~m}$ depth $(-0.769)$ on axis 1 and chlorophyll $a$ concentration (0.884) on axis 2 showing the highest correlations (Table 3 ). The canonical correspondence analysis triplots per type of tow showed 2 species-stations groups (Fig. 4a, b). Group 1 included only spring sampling stations in a productive environment (chlorophyll $a$ concentrations $=0.5-2.0 \mathrm{mg} \cdot \mathrm{m}^{-3}$ and zooplankton volumes $\left.=256-1,500 \mathrm{~mL} \cdot 1,000 \mathrm{~m}^{-3}\right)$ with low temperature $\left(16-18^{\circ} \mathrm{C}\right)$ and salinity $(33.2-33.6)$ in Subarctic Water. Group 2 included all autumn sampling stations and 2 additional spring sampling stations in oblique tows. In comparison with group 1, these stations were associated with a less productive (chlorophyll $a=0.5-1.0 \mathrm{mg} \cdot \mathrm{m}^{-3}$ and zooplankton volume $=1,024 \mathrm{~mL} \cdot 1,000 \mathrm{~m}^{-3}$ ), warmer $\left(18-22{ }^{\circ} \mathrm{C}\right)$, more saline (33.6-34.2) environment in different water masses (Subarctic Water, Transitional Water, and Subtropical Surface Water). At the surface, paralarvae of Onychoteuthis horstkottei (group 1) and of Abraliopsis sp. 1 and Abraliopsis sp. 2 (group 2) showed good correlations with chlorophyll $a$, high surface

Table 1. Ecological indexes of community structure by season and type of tow. PL, paralarvae.

Tabla 1. Índices ecológicos de la estructura de la comunidad por temporada y tipo de arrastre. PL, paralarvas.

\begin{tabular}{|c|c|c|c|c|}
\hline \multirow[b]{2}{*}{ Index } & \multicolumn{2}{|c|}{ Spring } & \multicolumn{2}{|c|}{ Autumn } \\
\hline & $\begin{array}{c}\text { Surface } \\
\text { tow }\end{array}$ & $\begin{array}{c}\text { Oblique } \\
\text { tow }\end{array}$ & $\begin{array}{c}\text { Surface } \\
\text { tow }\end{array}$ & $\begin{array}{c}\text { Oblique } \\
\text { tow }\end{array}$ \\
\hline Abundance $\left(\mathrm{PL} \cdot 1,000 \mathrm{~m}^{-3}\right)$ & 1,531 & 115 & 550 & 381 \\
\hline Richness (no. of species) & 5 & 9 & 8 & 17 \\
\hline Diversity (bits/ind.) & 0.694 & 1.040 & 1.352 & 1.609 \\
\hline Dominance & 0.909 & 0.201 & 0.222 & 0.232 \\
\hline
\end{tabular}

SD compuso el $95.1 \%$ y el $37.4 \%$ del total de la abundancia en los arrastres superficiales y oblicuos, respectivamente; en otoño, alcanzó el $32.5 \%$ en los arrastres superficiales y el $39.1 \%$ en los arrastres oblicuos. Abraliopsis sp. 1, registrada en otoño, alcanzó el 30.9\% del total de la abundancia en los arrastres superficiales y el $14.4 \%$ en los arrastres oblicuos.

\section{Análisis estadístico}

El análisis de agrupación para los arrastres superficiales mostró 2 grupos de especies-estaciones presentes únicamente en otoño en Agua Transicional y Agua Superficial Subtropical, uno caracterizado por la abundancia y frecuencia altas de Abraliopsis sp. 1 y el otro por la baja frecuencia de Argonauta sp. 3 y Japetella diaphana. Un tercer grupo caracterizado por el complejo SD se presentó en todas las masas de agua identificadas en primavera y otoño (Fig. 3a). La agrupación para los arrastres oblicuos mostró un patrón semejante. El primer grupo, compuesto principalmente por estaciones de muestreo de otoño, estuvo caracterizado por el complejo SD presente en las masas de agua cálida y en 3 estaciones con Agua Subártica. El pulpo pelágico J. diaphana y el calamar D. gigas formaron el segundo grupo, presente en Agua Transicional y Agua Subártica, y el tercer y el cuarto grupo incluyeron especies con baja frecuencia de aparición en ambas temporadas (Fig. 3b).

El análisis de correspondencia canónica para los arrastres superficiales explicó en sus primeros 2 ejes el 92.6\% de la varianza acumulada en la relación especies-ambiente. La concentración de clorofila $a(0.624)$ en el eje 1 y el volumen de zooplancton (0.899) en el eje 2 tuvieron la mejor correlación. De manera similar, para los arrastres oblicuos, la varianza acumulada explicó el $71.4 \%$ de la relación especies-ambiente, con el volumen de zooplancton a $10 \mathrm{~m}$ de profundidad $(-0.769)$ en el eje $1 \mathrm{y}$ la concentración de clorofila $a(0.884)$ en el eje 2 como las variables mejor correlacionadas (Tabla 3). Los gráficos del análisis de correspondencia canónica por tipo de arrastre muestran, cada uno, 2 grupos de especies-estaciones (Fig. 4a, b). El grupo 1 incluyó únicamente estaciones de muestreo de primavera en un ambiente productivo (clorofila $a=0.5-2.0 \mathrm{mg} \cdot \mathrm{m}^{-3} \mathrm{y}$ volumen de zooplancton $=256-1,500 \mathrm{~mL} \cdot 1,000 \mathrm{~m}^{-3}$ ) con baja temperatura $\left(16-18{ }^{\circ} \mathrm{C}\right)$ y salinidad $(33.2-33.6)$ distribuido en Agua Subártica. El grupo 2 incluyó todas las estaciones de muestreo de otoño y 2 adicionales de primavera en los arrastres oblicuos. En comparación con el grupo 1 , estas estuvieron relacionadas con un ambiente menos productivo (clorofila $a=0.5-1.0 \mathrm{mg} \cdot \mathrm{m}^{-3}$ y volumen de zooplancton $\left.=1,024 \mathrm{~mL} \cdot 1,000 \mathrm{~m}^{-3}\right)$, más cálido $\left(18-22^{\circ} \mathrm{C}\right)$ y más salino (33.6-34.2) localizado en diferentes masas de agua (Agua Subártica, Agua Transicional y Agua Superficial Subtropical). En superficie, las paralarvas de Onychoteuthis horstkottei (grupo 1) y de Abraliopsis sp. 1 y Abraliopsis sp. 2 (grupo 2) se correlacionaron bien con la clorofila $a$, la temperatura superficial alta y la baja concentración de clorofila $a$, 
Table 2. Acronym, abundance (number of paralarvae per 1,000 $\mathrm{m}^{3}$ ) by season and type of tow (surface, oblique), seasonal presence, and biogeographic affinity (Tm, temperate; $\mathrm{C}$, cosmopolite; Tr, tropical) of cephalopod paralarvae on the southwest coast of Baja California Sur. Tabla 2. Acrónimo, abundancia (número de paralarvas por cada 1,000 $\mathrm{m}^{3}$ ) por temporada y tipo de arrastre (superficial, oblicuo), presencia estacional y afinidad biogeográfica (Tm, templado; C, cosmopolita; Tr, tropical) de paralarvas de cefalópodos en la costa suroccidental de Baja California Sur.

\begin{tabular}{|c|c|c|c|c|c|c|c|c|c|c|}
\hline \multirow[b]{2}{*}{ Taxa } & \multirow[b]{2}{*}{ Acronym } & \multicolumn{4}{|c|}{ Abundance } & \multicolumn{2}{|c|}{$\begin{array}{l}\text { Seasonal } \\
\text { presence }\end{array}$} & \multicolumn{3}{|c|}{$\begin{array}{l}\text { Biogeographic } \\
\text { affinity }\end{array}$} \\
\hline & & Surface & Oblique & Surface & Oblique & Spring & Autumn & $\mathrm{Tm}$ & $\mathrm{C}$ & $\operatorname{Tr}$ \\
\hline \multicolumn{11}{|l|}{ Ancistrocheiridae } \\
\hline Ancistrocheirus cf. lesueurii & Anl & & & & 24 & & $\bullet$ & & $\bullet$ & \\
\hline \multicolumn{11}{|l|}{ Chiroteuthidae } \\
\hline \multicolumn{11}{|l|}{ Cranchiidae } \\
\hline Liguriella podophthalma & Lip & & 9 & & & $\bullet$ & & & $\bullet$ & \\
\hline \multicolumn{11}{|l|}{ Enoploteuthidae } \\
\hline Abraliopsis felis & Abf & & & & 4 & & $\bullet$ & $\bullet$ & & \\
\hline Abraliopsis sp. 1 & $\mathrm{Ab} 1$ & & & 170 & 55 & & $\bullet$ & & & $\bullet$ \\
\hline Gonatus sp. 1 & Go1 & & 5 & & & $\bullet$ & & $\bullet$ & & \\
\hline \multicolumn{11}{|l|}{ Ommastrephidae } \\
\hline Dosidicus gigas & Dog & 12 & 14 & 26 & & $\bullet$ & $\bullet$ & & & $\bullet$ \\
\hline Sthenoteuthis oualaniensis & Sto & & 12 & 11 & & $\bullet$ & $\bullet$ & & & $\bullet$ \\
\hline SD complex & $\mathrm{CSD}$ & 1,456 & 43 & 179 & 149 & $\bullet$ & $\bullet$ & & & $\bullet$ \\
\hline \multicolumn{11}{|l|}{ Onychoteuthidae } \\
\hline Onychoteuthis horstkottei & Onh & 37 & & & & $\bullet$ & & & & $\bullet$ \\
\hline Onychoteuthis spp. & On & & & & 12 & & $\bullet$ & & & \\
\hline \multicolumn{11}{|l|}{ Pyroteuthidae } \\
\hline Argonauta sp. 1 & Ar1 & & & & 4 & & $\bullet$ & & & $\bullet$ \\
\hline Argonauta sp. 3 & Ar3 & & & 10 & 9 & & $\bullet$ & & & $\bullet$ \\
\hline Argonauta sp. 4 & Ar4 & & & & 9 & & $\bullet$ & & & $\bullet$ \\
\hline \multicolumn{11}{|l|}{ Amphitretidae } \\
\hline Japetella diaphana & $\mathrm{Jad}$ & & 15 & 22 & 8 & $\bullet$ & $\bullet$ & & $\bullet$ & \\
\hline \multicolumn{11}{|l|}{ Octopodidae } \\
\hline Octopus hubbsorum & Och & 10 & & & & $\bullet$ & & & & $\bullet$ \\
\hline Octopus rubescens & Ocr & & 3 & & & $\bullet$ & & $\bullet$ & & \\
\hline Octopus veligero & Ocv & & 12 & & & $\bullet$ & & & & $\bullet$ \\
\hline Octopus sp. 1 & Oc1 & & & & 3 & & $\bullet$ & & & $\bullet$ \\
\hline Octopus sp. 2 & Oc2 & & & & 4 & & $\bullet$ & & & $\bullet$ \\
\hline Octopus sp. 3 & Oc3 & 16 & 2 & & 4 & $\bullet$ & $\bullet$ & & & $\bullet$ \\
\hline Octopus spp. & Oc & & & 13 & 4 & & $\bullet$ & & & \\
\hline Total & & 1,531 & 115 & 550 & 381 & 10 & 18 & 3 & 4 & 17 \\
\hline
\end{tabular}


a
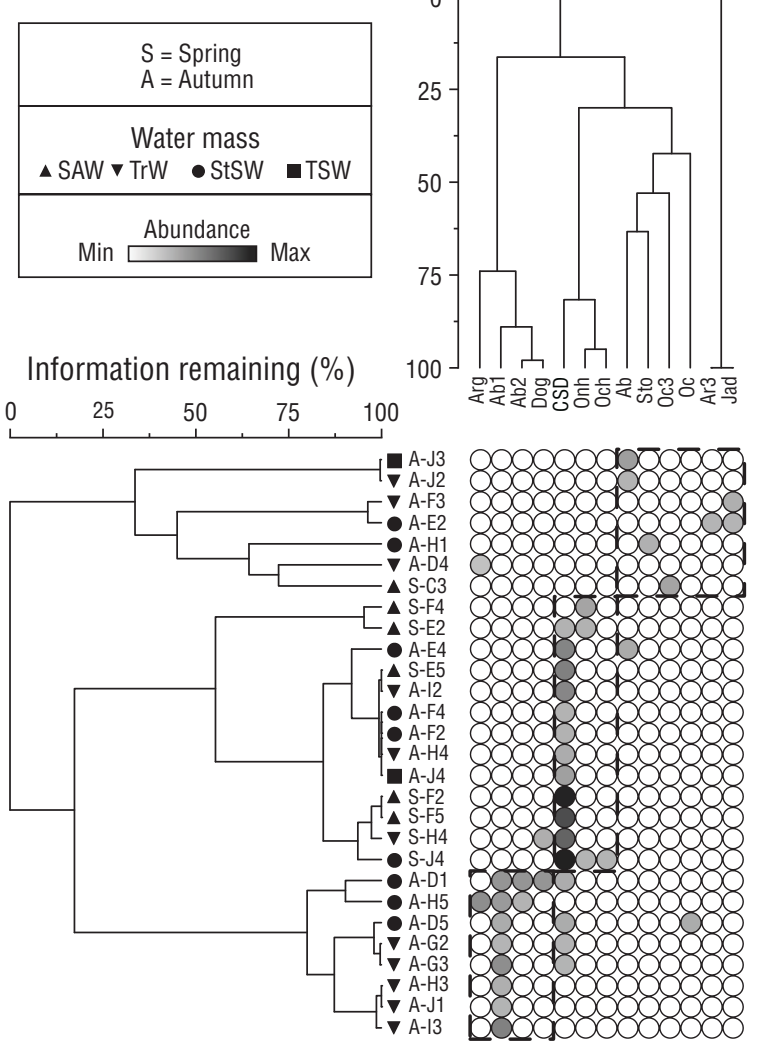

b
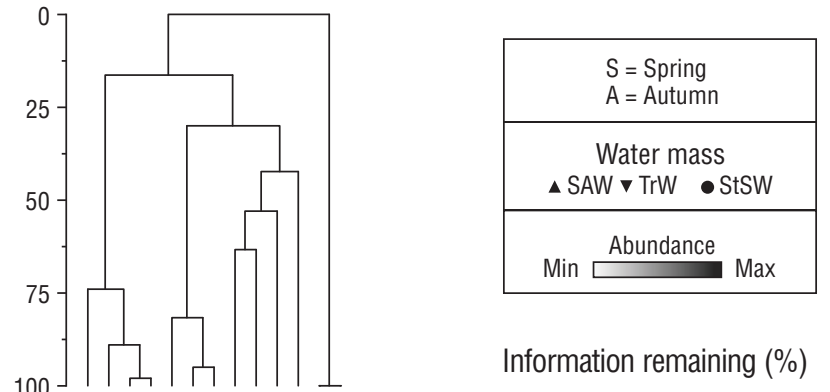

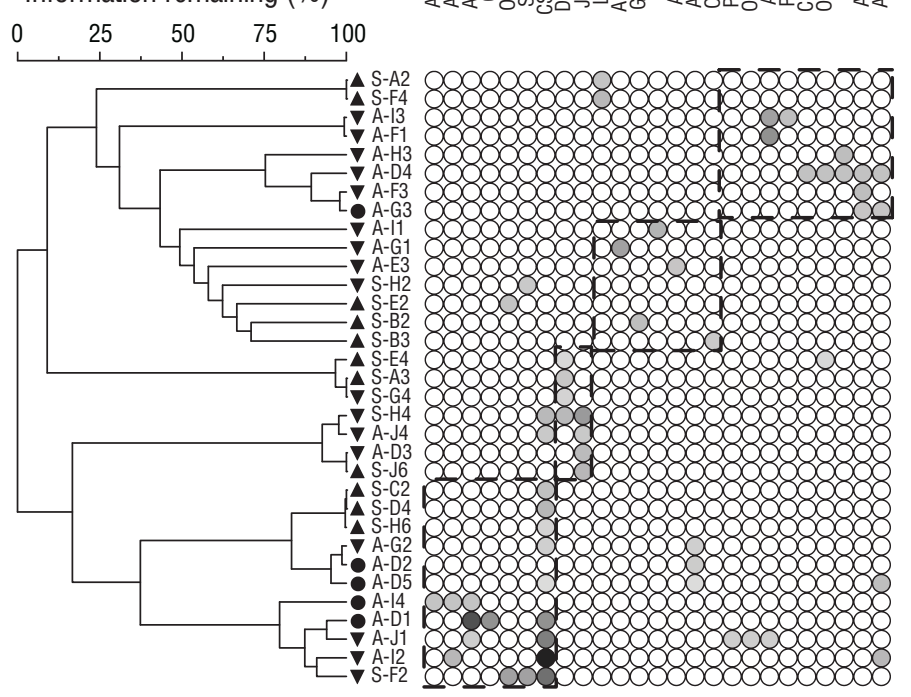

Figure 3. Two-way cluster analysis for surface (a) and oblique (b) tows. Water masses: SAW, Subarctic Water; TrW, Transitional Water; StSW, Subtropical Surface Water; TSW, Tropical Surface Water. Sampling station code is noted after the hyphen. For species acronyms, see Table 1. Rectangles with broken lines indicate the species-stations groups.

Figura 3. Análisis de agrupamiento de 2 vías para los arrastres superficiales (a) y oblicuos (b). Masas de agua: SAW, Agua Subártica; TrW, Agua Transicional; StSW, Agua Superficial Subtropical; TSW, Agua Superficial Tropical. Después del guion se indica el código de la estación de muestreo. Para los acrónimos de las especies, ver Tabla 1. Los rectángulos con líneas discontinuas indican los grupos de especies-estaciones.

temperature, and low chlorophyll a concentration, respectively. In oblique tows, Octopus veligero (group 1) correlated with chlorophyll $a$, while 3 species from the genus Argonauta correlated with low zooplankton volumes and high temperature (Fig. 4a, b).

\section{Discussion}

This is the first time the analysis of seasonal changes in the community of cephalopod paralarvae off the SWC under normal conditions (without ENSO) is presented. The analysis of 2 types of simultaneous tows showed substantial differences in the type of information that is obtained from each tow and their utility.

Integral studies (abundance-environment) of cephalopod paralarvae in areas of high biological productivity, such as upwelling systems, have increased worldwide due to the growing commercial demand for fishery resources. This study, conducted on the SWC during 2003, a year with no respectivamente. En los arrastres oblicuos, Octopus veligero (grupo 1) se correlacionó con la clorofila $a$, mientras que 3 especies del género Argonauta se correlacionaron con los volúmenes de zooplancton bajos y la temperatura alta (Fig. 4a, b).

\section{Discusión}

Se presenta, por primera vez, el análisis del cambio estacional en la comunidad de paralarvas de cefalópodos frente a la CSO en condiciones normales ( $\sin$ ENOS). El análisis del uso de 2 tipos de arrastre simultáneos muestra que existen diferencias sustanciales en el tipo de información que se obtiene de cada uno, así como en su utilidad.

A nivel mundial, el estudio integral (abundancia-ambiente) de las paralarvas de cefalópodos en áreas de alta productividad biológica, como los sistemas de surgencias, se ha incrementado debido a la creciente demanda comercial de recursos de interés pesquero. Este estudio, realizado en la CSO durante 
anomalous ENSO-induced thermal conditions (http://www. cpc.ncep.noaa.gov/products/analysis_monitoring/ensostuff/ensoyears.shtml), allowed us to determine, for the first time, the influence of seasonal changes on the community of cephalopod paralarvae in the southernmost region of the California Current upwelling system.

Durazo (2015) determined that the region to the north of Point Eugenia $\left(28^{\circ} \mathrm{N}\right)$ was homogeneous, with low temperature and salinity during winter and spring and predominating subarctic conditions throughout the year, whereas to the south subarctic conditions were predominant in winter and spring and tropical-subtropical conditions in summer and autumn. In the region to the north of our study area, anomalous intrusions of warm and cool water during the 1997-2001 ENSO event prompted drastic changes in different marine communities (e.g., fish and zooplankton larvae and adults, including paralarvae) (Lea and Rosenblatt 2000, Lavaniegos et al. 2002, Granados-Amores et al. 2010, Jiménez-Rosenberg et al. 2010).

Granados-Amores et al. (2010) indicated that during the 1997-1998 ENSO event, the northward extension of paralarvae of tropical species reached the region off Punta Baja, Baja California (Camarillo-Coop 2006, Granados-Amores et al. 2010). During the El Niño-La Niña transition, temperate species showed a 2-fold abundance increase with distribution in Subarctic Water, and tropical species showed a $50 \%$ abundance decrease with distribution in the warm water mass to the south; the continued presence of Subarctic Water from Ensenada to the south of Point Abreojos, in the period from autumn 1998 to late
2003, un año sin condiciones térmicas anómalas por efecto del ENOS (http://www.cpc.ncep.noaa.gov/products/analysis monitoring/ensostuff/ensoyears.shtml), permitió establecer, por primera vez, la influencia del cambio estacional sobre la comunidad de paralarvas de cefalópodos en la región más sureña del sistema de surgencias de la corriente de California.

Durazo (2015) estableció que al norte de punta Eugenia $\left(28^{\circ} \mathrm{N}\right)$, la región es homogénea con temperatura y salinidad bajas durante invierno y primavera y dominio subártico todo el año, mientras que al sur existe una alternancia entre el dominio subártico en invierno y primavera y el dominio tropical-subtropical en verano y otoño. En la región al norte de nuestra área de estudio, las invasiones anómalas de agua cálida y fría durante el evento ENOS de 1997-2001 promovieron un cambio drástico en diferentes comunidades marinas (e.g., de larvas y adultos de peces y del zooplancton, incluidas las paralarvas) (Lea y Rosenblatt 2000, Lavaniegos et al. 2002, Granados-Amores et al. 2010, Jiménez-Rosenberg et al. 2010).

Granados-Amores et al. (2010) destacaron que durante el evento ENOS de 1997-1998, el avance al norte de paralarvas de especies tropicales alcanzó la región frente a punta Baja, Baja California (Camarillo-Coop 2006, Granados-Amores et al. 2010). Durante la transición de El Niño a La Niña, las especies templadas duplicaron su abundancia relativa distribuyéndose en Agua Subártica y las especies tropicales redujeron su abundancia en un 50\% distribuyéndose al sur en la masa de agua cálida; la posterior permanencia de Agua Subártica desde Ensenada hasta el sur de punta Abreojos, desde el otoño de 1998 hasta finales del verano de 1999, determinó

Table 3. Summary of the canonical correspondence analysis applied to abundance and environmental data from surface and oblique tows. Significant correlations for surface tows: axis $1, F=1.511, P<0.590$; remaining axes, $F=0.656, P<0.854$. Significant correlations for oblique tows: axis $1, F=1.500, P<0.344$; remaining axes, $F=0.995, P<0.444$.

Tabla 3. Resumen del análisis de correspondencia canónica aplicado a los datos de abundancia y datos ambientales obtenidos de los arrastres superficiales y oblicuos. Correlaciones significativas para los arrastres superficiales: eje $1, F=1.511, P<0.590$; resto de los ejes, $F=0.656, P<0.854$. Correlaciones significativas para los arrastres oblicuos: eje $1, F=1.500, P<0.344$; resto de los ejes, $F=0.995, P<0.444$.

\begin{tabular}{|c|c|c|c|c|c|c|}
\hline & \multicolumn{3}{|c|}{ Surface } & \multicolumn{3}{|c|}{ Oblique } \\
\hline & Axis & Axis 2 & Axis 3 & Axis & Axis 2 & Axis 3 \\
\hline Eigenvalues & 0.366 & 0.100 & 0.032 & 0.568 & 0.481 & 0.301 \\
\hline Species-environment correlations & 0.712 & 0.380 & 0.251 & 0.853 & 0.823 & 0.694 \\
\hline \multicolumn{7}{|l|}{ Cumulative variance (\%) } \\
\hline Species data & 11.3 & 14.4 & 15.4 & 8.9 & 16.5 & 21.2 \\
\hline Species-environment relation & 72.7 & 92.6 & 99.0 & 38.7 & 71.4 & 92.0 \\
\hline \multicolumn{7}{|l|}{ Environmental variables } \\
\hline Temperature & -0.464 & 0.325 & -0.664 & -0.342 & -0.146 & -0.121 \\
\hline Salinity & 0.128 & 0.200 & -0.761 & -0.220 & 0.159 & -0.179 \\
\hline Zooplankton volume & 0.498 & 0.899 & 0.163 & -0.769 & 0.462 & -0.384 \\
\hline Chlorophyll $a$ & 0.624 & 0.034 & 0.471 & -0.070 & 0.884 & 0.461 \\
\hline
\end{tabular}



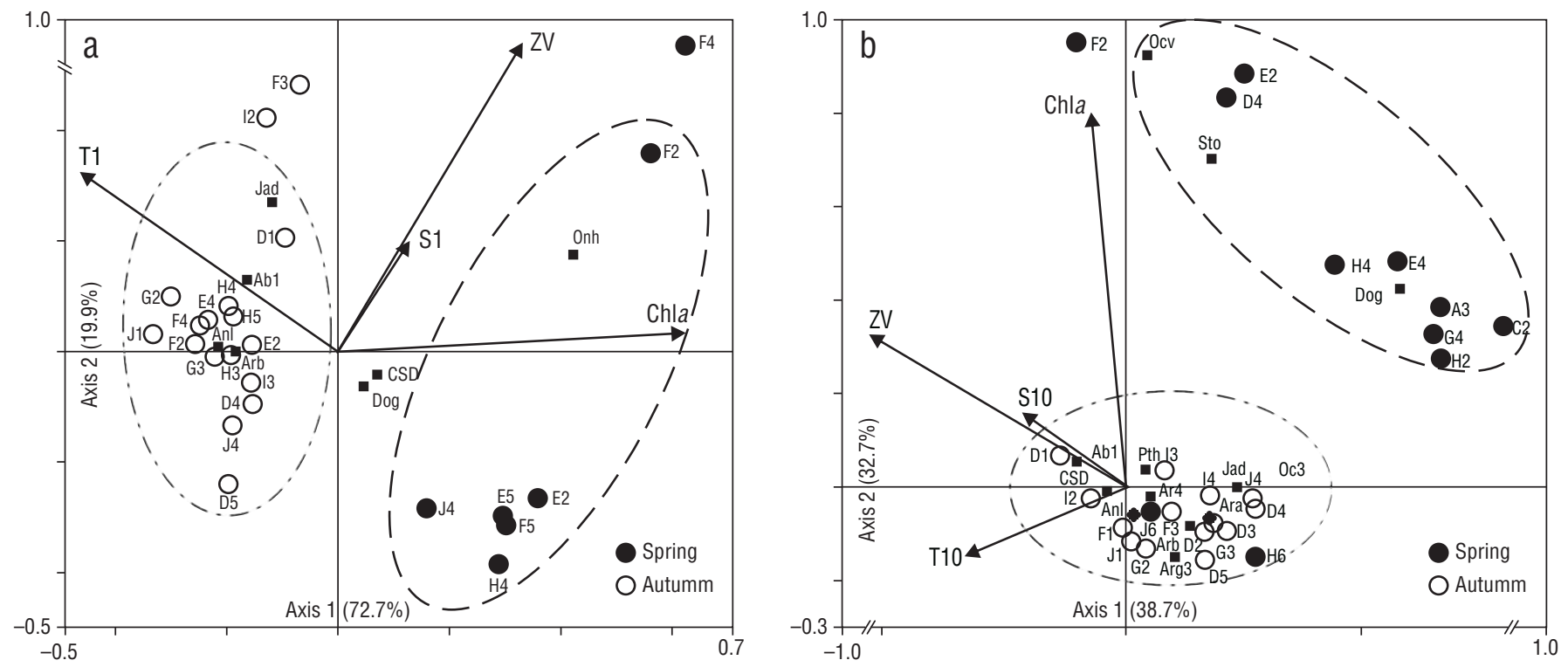

Figure 4. Triplots from the canonical correspondence analysis of surface (a) and oblique tows (b). Environmental variables: ZV, zooplankton volume; Chla, chlorophyll $a$; T1, temperature at $1 \mathrm{~m}$ depth; S1, salinity at $1 \mathrm{~m}$ depth; T10, temperature at $10 \mathrm{~m}$ depth; S10, salinity at $10 \mathrm{~m}$ depth. For species acronyms, see Table 1. Letters A-J followed by numbers indicate the sampling station after the corresponding symbol.

Figura 4. Gráficas del análisis de correspondencia canónica para los arrastres superficiales (a) y oblicuos (b). Variables ambientales: ZV, volumen de zooplancton; Chla, concentración de clorofila $a$; T1, temperatura a $1 \mathrm{~m}$ de profundidad; S1, salinidad a $1 \mathrm{~m}$ de profundidad; T10, temperatura a $10 \mathrm{~m}$ de profundidad; S10, salinidad a $10 \mathrm{~m}$ de profundidad. Para los acrónimos de las especies, ver Tabla 1. Las letras A-J seguidas de números después de los símbolos correspondientes indican las estaciones de muestreo.

summer 1999, determined de disappearance of taxa with tropical-subtropical and cosmopolite affinities, giving rise to a temperate community dominated by the Gonatidae family (Granados-Amores et al. 2010). In comparison, the seasonal change with no ENSO effects off the SWC were significantly different. Though samples were collected in seasons with contrasting oceanographic conditions (Durazo 2015), the biogeographical affinity of paralarvae was predominantly tropical-subtropical and the greatest change was given not by the dominant biogeographical affinity or by taxon distribution in a particular water mass, as reported for the north, but by the restructuring of the community as richness of tropical taxa almost doubled in autumn when Subarctic Water retreated and the warm environment expanded with the appearance of 3 water masses: Tropical Surface Water, Subtropical Surface Water, and Equatorial Subsurface Water (100-225 m) (Avendaño-Ibarra et al. 2010). The change in community structure in autumn was also modulated by the decrease in the abundance of paralarvae, in particular of the SD complex (dominant taxon), which together with the arrival of new species to the region, prompted an increase in diversity and a decrease in dominance. Avendaño-Ibarra et al. (2010) analyzed the same survey data examined in the present study and recorded a significant increase in the number and abundance of fish larvae of tropical species during autumn, similar to our results; however, fish larval abundances were 3 times higher in autumn and paralarval abundance decreased during this season. la desaparición de taxones de afinidad tropical-subtropical y cosmopolita y la aparición de una comunidad templada dominada por la familia Gonatidae (Granados-Amores et al. 2010). A modo de comparación, el cambio estacional sin efecto ENOS frente a la CSO fue significativamente diferente. Aunque las muestras se recolectaron en temporadas oceanográficamente contrastantes (Durazo 2015), la afinidad biogeográfica de las paralarvas se mantuvo predominantemente tropical-subtropical, y el mayor cambio no fue en la afinidad biogeográfica dominante o en la distribución de los taxones en una masa de agua en particular, como lo reportado para el norte, sino en la reestructuración de la comunidad al incorporarse casi el doble de la riqueza de taxones tropicales en otoño por efecto de la disminución de Agua Subártica y de la expansión del ambiente cálido debido a la llegada a la zona de 3 masas de agua cálida: la Superficial Tropical, la Superficial Subtropical y la Subsuperficial Ecuatorial (100-225 m) (Avendaño-Ibarra et al. 2010). El cambio en la estructura de la comunidad en otoño también estuvo modulado por la disminución en la abundancia de paralarvas, particularmente del complejo SD (taxón dominante), que junto con la llegada de nuevas especies a la región, promovió un incremento en la diversidad y una disminución en la dominancia. Avendaño-Ibarra et al. (2010) analizaron los mismos datos de muestreo que el presente estudio y registraron un incremento significativo en el número y abundancia de larvas de peces de especies tropicales durante otoño, semejante a nuestros resultados, pero la abundancia de larvas de peces fue 
The latitudinal movement of water masses and the formation of cephalopod paralarval assemblages on the SWC were also observed in the Gulf of California during 2004-2007 when there were no significant ENSO effects (De Silva-Dávila et al. 2015). In the Gulf of California there were 3 associations that were determined by temperature and salinity, but also by zooplankton volumes and chlorophyll $a$ concentrations, as was observed on the SWC. These 3 associations showed latitudinal boundaries that changed with the extension or retrieval of surface water masses that were present year-round (De Silva-Dávila et al. 2015). On the SWC the groups determined by the canonical correspondence analysis were associated with the 2 predominant environments: the productive cool environment in spring and the less productive warm environment in autumn. However, most taxa (group 2) were not exclusively distributed in a single water mass, indicating the absence of correlations with temperature and salinity, and the characteristic oceanographic variability with typically 3 warm water masses, a high variability compared to that recorded for the region off the central portion of the peninsula and further north (Lynn and Simpson 1987, De Silva-Dávila et al. 2002). A wide variety of individual species responses to environmental seasonality was expected because every species or group of species (neritic, oceanic) has different reproductive strategies (Moreno et al. 2009). In tropical areas with only one warm surface water mass, such as the Gulf of Tehuantepec, there were no recorded changes in the community of paralarvae and their segregation was the result of mesoscale processes (Aceves-Medina et al. 2017).

The species that characterized each group in the SWC were different from the ones reported for the Gulf of California. Onychoteuthis horstkottei (group 1, surface tow) is distributed in the tropical equatorial Pacific (Bolstad 2010). However, O. horstkottei paralarvae have been recorded in warm environments from the Gulf of California to the Gulf of Tehuantepec (De Silva-Dávila et al. 2015, Aceves-Medina et al. 2017). Their presence in a cold environment on the SWC in spring could be associated with their northern geographic range limit or with a broader geographic range than the previously reported. The same goes for paralarvae of Abraliopsis sp. 1 and Abraliopsis sp. 2 (group 2, surface tow), probably corresponding to Abraliopsis falco and Abraliopsis affinis, respectively (Jereb and Roper 2010). Octopus veligero (group 1, oblique tow) is an octopus species that, in the adult stage, is well known to be distributed in waters off the region from Point Eugenia to the tip of the Baja California Peninsula and into La Paz Bay, in the Gulf of California (Jereb et al. 2014). Its paralarvae, on the other hand, have been recorded in the Gulf of Tehuantepec (at 29-34 ${ }^{\circ} \mathrm{C}$ and $33.1-34.4$ salinity) (Alejo-Plata et al. 2013). Despite the large number of zooplankton surveys on the Pacific coasts of Mexico (Granados-Amores et al. 2010, De Silva-Dávila et al. 2015, Aceves-Medina et al. 2017), our study on the SWC was the first to report the presence
3 veces mayor en otoño, a diferencia de las paralarvas cuya abundancia disminuyó en esta temporada.

$\mathrm{El}$ avance latitudinal en las masas de agua y la formación de grupos de paralarvas de cefalópodos observados en la CSO tuvieron un comportamiento semejante al registrado para el golfo de California durante el periodo 2004-2007, también sin efecto significativo de ENOS (De Silva-Dávila et al. 2015). En el golfo de California se registraron 3 asociaciones determinadas por la temperatura y la salinidad, pero también por los volúmenes de zooplancton y las concentraciones de clorofila $a$, como en la CSO. Las 3 asociaciones presentaron límites latitudinales que se modificaron con el avance o retroceso de las masas de agua superficiales presentes a lo largo del año (De Silva-Dávila et al. 2015). En la CSO, los grupos obtenidos a partir del análisis de correspondencia canónica se relacionaron con los 2 ambientes predominantes: el productivo y frío de primavera y el poco productivo y cálido de otoño. Sin embargo, la gran mayoría de los taxones (grupo 2) no se distribuyó en una única masa de agua, lo cual refleja la ausencia de correlaciones con la temperatura y salinidad, así como la presencia característica de una variabilidad oceanográfica con típicamente 3 masas de agua cálidas, que es alta comparada con la registrada en la región central de la península y más al norte (Lynn y Simpson 1987, De Silva-Dávila et al. 2002). Una amplia variedad de respuestas individuales de las especies a la estacionalidad del medio es de esperarse, debido a que cada especie o grupos de especies (neríticas, oceánicas) presentan estrategias reproductivas diferentes (Moreno et al. 2009). En áreas tropicales con una sola masa de agua cálida superficial, como el golfo de Tehuantepec, no se registró un cambio en la comunidad de paralarvas y su segregación estuvo dada por la presencia de procesos de mesoescala (Aceves-Medina et al. 2017).

Las especies características de cada grupo en la CSO fueron diferentes a las registradas en el golfo de California. Onychoteuthis horstkottei (grupo 1, arrastre superficial) se distribuye en el Pacífico ecuatorial tropical (Bolstad 2010). Sin embargo, sus paralarvas han sido registradas desde el golfo de California hasta el golfo de Tehuantepec en ambientes cálidos (De Silva-Dávila et al. 2015, Aceves-Medina et al. 2017). Su presencia en la CSO en un ambiente frío en primavera pudiera relacionarse con su límite norte de distribución geográfica o puede evidenciar una distribución más amplia de la actualmente registrada. Lo mismo aplica para las paralarvas de Abraliopsis sp. 1 y Abraliopsis sp. 2 (grupo 2, arrastre superficial), probablemente correspondientes a Abraliopsis falco y Abraliopsis affinis, respectivamente (Jereb y Roper 2010). Octopus veligero (grupo 1, arrastre oblicuo) es una especie de pulpo cuyos adultos tienen una distribución conocida en aguas frente a la región desde punta Eugenia hasta la punta de la península de Baja California y dentro de bahía de La Paz, en el golfo de California (Jereb et al. 2014). Por otro lado, sus paralarvas se han registrado en el golfo de Tehuantepec (a 29-34 ${ }^{\circ} \mathrm{C}$ y salinidad de 33.1-34.4) (Alejo-Plata et al. 2013). A pesar del amplio número de muestreos de zooplancton 
of this species in Subarctic Water off northwestern Mexico. This first report confirms the known geographic range of this species and provides information on the species biology and ecology, which is mostly unknown (Jereb et al. 2014). With respect to paralarvae of the genus Argonauta (group 2 , oblique tow), which were recorded only in autumn, its biogeographic affinity (pantropical-oceanic) (Nesis 2003) and high abundance in the southern portion of the Gulf of California $\left(23-26{ }^{\circ} \mathrm{C}\right)$ (De Silva-Dávila 2013) were associated with increasing temperatures during this season. On the other hand, paralarvae of the SD complex, which characterized the tropical association in the Gulf of California, were widely distributed on the SWC in both seasons but showed no significant correlation with any environmental variable, nor did they characterize a specific group.

In the early life stages, paralarvae of some species are associated with productive zones, where chlorophyll $a$ concentrations and zooplankton volumes provide large nutrient quantities for development (González et al. 2005, Moreno et al. 2009, Vidal et al. 2010), suggesting the coupling of species to regional productivity pulses (De Silva-Dávila et al. 2015, Aceves-Medina et al. 2017). Though chlorophyll $a$ concentrations have been associated with paralarval abundance, most paralarvae, however, do not directly feed on phytoplankton because they become voracious predators soon after hatching (Chen et al. 1996). In particular, hatched Ommastrephidae paralarvae, including the SD complex, have a typical rhynchoteuthion shape with fused tentacles that form a proboscis that is not useful for capturing prey until it separates, hindering food capture (O'Dor et al. 1986, Shea 2005). The mucus on the bodies of paralarvae ( $<4.0 \mathrm{~mm} \mathrm{ML}$ ) allows growth of microorganisms, which paralarvae can collect and ingest with their proboscis (Vidal and Haimovici 1998) and are probably their main source of energy during this stage of development. After the proboscis separates, paralarvae are considered juveniles and can more efficiently make use of the available zooplankton for their development (Shea 2005, De Silva-Dávila 2013), since they can then feed on calanoid copepods and on larger and more diverse prey species (amphipods, euphausiids, cephalopods, and fish) as they grow (Vidal and Haimovici 1998, Camarillo-Coop et al. 2013). Zooplankton volumes could thus be used as reliable indicators of the food available for paralarvae.

The California Current upwelling system off the Baja California Peninsula has all the necessary features to cover the energy requirements of highly voracious organisms such as squid. To the north of Point Abreojos and further north to Ensenada, zooplankton volumes recorded during the 19972001 ENSO event were typical of the region, in spite of the anomalous warm conditions (Lavaniegos et al. 2002). By contrast, zooplankton volumes in the spring and autumn oblique tows were higher during normal conditions on the SWC (Table 4). Both maximum total abundance of paralarvae and the abundance of the SD complex during the ENSO transition realizados a lo largo de las costas mexicanas del Pacífico (Granados-Amores et al. 2010, De Silva-Dávila et al. 2015, Aceves-Medina et al. 2017), nuestro estudio en la CSO registra por primera vez las paralarvas de esta especie en Agua Subártica frente a la zona noroeste de México. Este primer registro refleja bien la distribución conocida de la especie, y los resultados obtenidos aportan información sobre su biología y ecología, que se desconocen en su mayoría (Jereb et al. 2014). En cuanto a las paralarvas del género Argonauta (grupo 2, arrastre oblicuo), registrado únicamente en otoño, su afinidad pantropical oceánica (Nesis 2003) y su elevada abundancia en el sur del golfo de California $\left(23-26^{\circ} \mathrm{C}\right.$ ) (De Silva-Dávila 2013) se relacionaron con el incremento de la temperatura en la temporada. Por otra parte, las paralarvas del complejo $\mathrm{SD}$, que caracterizaron la asociación tropical en el golfo de California, se distribuyeron ampliamente en la CSO en ambas temporadas, pero no mostraron una correlación significativa con alguna variable ambiental ni caracterizaron algún grupo.

En las etapas tempranas de vida, las paralarvas de algunas especies se asocian a zonas productivas, donde las concentraciones de clorofila $a$ y los volúmenes de zooplancton determinan una alta disponibilidad de alimento para su desarrollo (González et al. 2005, Moreno et al. 2009, Vidal et al. 2010) y sugieren un acoplamiento de las especies a los pulsos de productividad regional (De Silva-Dávila et al. 2015, Aceves-Medina et al. 2017). Sin embargo, aunque la concentración de clorofila $a$ ha sido relacionada con la abundancia de paralarvas, la mayoría de ellas no se alimentan directamente del fitoplancton ya que son depredadoras voraces desde la eclosión (Chen et al. 1996). En particular, las paralarvas de ommastréfidos, incluso el complejo SD, eclosionan con la típica forma rhynchoteuthion que presenta los tentáculos fusionados en una forma de probóscide que no es funcional para la captura de presas hasta que se separa, lo que dificulta la captura de alimento (O’Dor et al. 1986, Shea 2005). También se sabe que el mucus que recubre el cuerpo de las paralarvas $(<4.0 \mathrm{~mm} \mathrm{LM})$ permite el crecimiento de microrganismos, que con ayuda de la probóscide pueden recolectar e ingerir (Vidal y Haimovici 1998) y que, probablemente, son la principal fuente de energía en esta fase del desarrollo. A partir de la separación de la probóscide, los organismos se consideran juveniles y pueden aprovechar con mayor eficiencia el zooplancton disponible para su crecimiento (Shea 2005, De Silva-Dávila 2013) al alimentarse de copépodos calanoideos y al ampliar el tamaño y diversidad de sus presas (anfípodos, eufáusidos, cefalópodos y peces) conforme aumentan de talla (Vidal y Haimovici 1998, Camarillo-Coop et al. 2013). En consecuencia, los volúmenes de zooplancton pudieran representar un buen indicador de alimento disponible para las paralarvas.

El sistema de surgencias de la corriente de California frente a la península de Baja California cumple con las características para cubrir los requerimientos energéticos de organismos altamente voraces como los calamares. Al norte de punta Abreojos y hasta Ensenada, durante el evento ENOS de 1997-2001 se registraron volúmenes de zooplancton que, 
phase (July 1998) were much higher on the northwestern coast than on the SWC, but the average values obtained with only 2 surveys for both coasts were very similar. This suggests that during the 1997-1998 period the anomalous warming of the system was more favorable than were zooplankton volumes to the presence of highly abundant paralarvae, particularly paralarvae of the Ommastrephidae family (D. gigas, S. oualaniensis, and the SD complex), which dominated the community (Camarillo-Coop 2006). The high abundance of the SD complex that brought on a spawning event off Point Eugenia when conditions were anomalously warm is evidence of the reproductive plasticity of these species and their opportunistic nature (Camarillo-Coop 2006, Hoving et al. 2013, Hernández-Muñoz et al. 2016). In the Gulf of California, zooplankton volumes (oblique tows) were mostly higher than those recorded for the northwestern coast and the SWC, but total paralarval abundance in May (spring) was 1.5 and 4.8 times higher than maximum total abundances for the northwestern coast and SWC, respectively (Table 4a). With respect to paralarvae of the SD complex in May, the number of paralarvae in the gulf nearly reached the maximum number recorded for the northwestern coast (transition phase) and was twice the maximum number recorded for the SWC (Table 4a).

On the other hand, the paralarvae of the SD complex collected in the surface tows were significantly more abundant than those collected in the oblique tows on the SWC, with 8.5 times more paralarvae for this taxon (Table 4). The same thing occurred in the Gulf of California. The abundances obtained from cruises that carried out both types of tows (S-195 and S-207) showed 8.6 times more paralarvae of this complex in surface tows (Table 4). The average value $\left(817 \mathrm{PL} \cdot 1,000 \mathrm{~m}^{-3}\right)$ for paralarvae collected with surface tows on the SWC is the second highest recorded for the region off northwestern Mexico, after the average for the Gulf of California. This result is important because D. gigas, which is part of this complex, supports the most important cephalopod fishery on the Pacific coast of Mexico, with landings of 36,381 metric tons in 2013 (CONAPESCA 2014, Zepeda-Benites et al. 2017). In spite of this, there is no sampling program for obtaining the necessary data to identify the species hatching areas and seasonal patterns. The presence of naturally deposited egg masses of $D$. gigas at less than $20 \mathrm{~m}$ depth when temperature was $24-27{ }^{\circ} \mathrm{C}$ (Staaf et al. 2008, Birk et al. 2017) and the efficiency of surface tows for catching paralarvae of the SD complex in the tropical equatorial Pacific (Staaf et al. 2013), the Gulf of California (De Silva-Dávila 2013), and waters on the SWC (this study) prove that this type of tow, which is of low cost and easily executed in short operation times, could be the best sampling option to start a monitoring and assessment program for squid off northwestern Mexico. The recorded differences when surface and oblique tows are analyzed separately could lead to different inferences and conclusions on the entire community of paralarvae. a pesar del calentamiento anómalo, estuvieron dentro de los valores típicos para la región (Lavaniegos et al. 2002). Como comparación, en condiciones normales en la CSO, los volúmenes de zooplancton recolectados en los arrastres oblicuos de primavera y otoño fueron más elevados (Tabla 4). En cambio, la abundancia total máxima de paralarvas registrada en la fase de transición de ENOS (julio de 1998) y la abundancia del complejo SD fueron mucho mayores en la costa noroccidental que en la CSO, aunque los promedios para ambas con solo 2 muestreos fueron muy semejantes. Lo anterior sugiere que durante el periodo 1997-1998, el calentamiento anómalo del sistema favoreció más que los volúmenes de zooplancton a la presencia de alta abundancia de paralarvas, especialmente de la familia Ommastrephidae (D. gigas, S. oualaniensis y el complejo SD), que dominó la comunidad (Camarillo-Coop 2006). En particular, la alta abundancia del complejo SD que estableció la presencia de un evento de desove frente a punta Eugenia en condiciones anómalamente cálidas para la región evidencia la plasticidad reproductiva de estas especies y su naturaleza oportunista (Camarillo-Coop 2006, Hoving et al. 2013, Hernández-Muñoz et al. 2016). En el golfo de California los volúmenes de zooplancton (arrastres oblicuos), fueron en su mayoría más altos que los registrados en la costa noroccidental y en la CSO, pero resalta la abundancia total de paralarvas de mayo (primavera), que fue 1.5 y 4.8 veces mayor que las abundancias totales máximas registradas en la costa noroccidental y CSO, respectivamente (Tabla 4a). En cuanto a las paralarvas del complejo SD registradas también en mayo, las del golfo casi alcanzaron el número máximo registrado en la costa noroccidental (fase de transición) y fueron el doble del máximo registrado en la CSO (Tabla 4a).

Por otra parte, las paralarvas del complejo SD recolectadas en los arrastres superficiales fueron significativamente más abundantes que las de los oblicuos realizados en la CSO, con 8.5 veces más paralarvas promedio para este taxón (Tabla 4). En el golfo de California, sucedió lo mismo. Las abundancias de los cruceros en los que se realizaron ambos tipos de arrastre (S-195 y S-207) mostraron que en superficie se recolectaron 8.6 veces más paralarvas de este complejo (Tabla 4). El valor promedio $\left(817 \mathrm{PL} \cdot 1,000 \mathrm{~m}^{-3}\right)$ de paralarvas recolectadas con arrastres superficiales en la CSO es el segundo mayor registrado para la zona noroeste de México, después del promedio para el golfo de California. La importancia de este resultado radica en que $D$. gigas, que forma parte de este complejo de especies, soporta la pesquería de cefalópodos más importante en las costas mexicanas del Pacífico con un desembarque de 36,381 toneladas métricas en 2013 (CONAPESCA 2014, Zepeda-Benites et al. 2017). A pesar de lo anterior, no existe un programa de muestreo que permita la identificación de las áreas de eclosión y los patrones temporales de esta especie. La presencia de masas de huevos de D. gigas en estado natural localizadas a menos de $20 \mathrm{~m}$ de profundidad cuando la temperatura fue de $24-27^{\circ} \mathrm{C}$ (Staaf et al. 2008, Birk et al. 2017) y la eficiencia de los arrastres superficiales en la captura de paralarvas del complejo SD, tanto en el Pacífico 
Table 4. Zooplankton volume (ZV), total paralarval abundance, and abundance of the Sthenoteuthis oualaniensis-Dosidicus gigas complex (SDC) in 2 types of tows: oblique tow (a) and surface tow (b). Samples were collected during the El Niño, transition, and La Niña phases of the 1997-2001 ENSO event on the northwest coast of Mexico (taken from Lavaniegos et al. 2002, Camarillo-Coop 2006); during 2003 on the southwestern coast of Baja California (this study, grey shade); and during the 2004-2007 period in the Gulf of California (taken from De Silva-Dávila 2013). Tran = El Niño-La Niña transition; PL = paralarvae. ${ }^{*}$ Cruises with both types of tows.

Tabla 4. Volumen de zooplancton (ZV) y abundancia total de paralarvas y del complejo Sthenoteuthis oualaniensis-Dosidicus gigas (SDC) en 2 tipos de arrastre: (a) arrastre oblicuo y (b) arrastre superficial. Se muestran datos de muestreos realizados durante las fases El Niño, transición y La Niña del evento ENOS de 1997-2001 en la costa noroccidental de México (tomados de Lavaniegos et al. 2002, CamarilloCoop 2006); durante 2003 en la costa suroccidental de Baja California (este estudio, sombreado en gris) y durante el periodo $2004-2007$ en el golfo de California (De Silva-Dávila 2013). Tran = transición El Niño-La Niña; PL = paralarvas. *Cruceros con ambos tipos de arrastre.

(a)

\begin{tabular}{|c|c|c|c|c|c|c|c|}
\hline \multirow[b]{2}{*}{ Cruise } & \multirow[b]{2}{*}{ Date } & \multicolumn{4}{|c|}{$\begin{array}{c}\text { Mean ZV } \\
\left(\mathrm{mL} \cdot 1,000 \mathrm{~m}^{-3}\right)\end{array}$} & \multicolumn{2}{|c|}{$\begin{array}{c}\text { Abundance } \\
\left(\mathrm{PL} \cdot 1,000 \mathrm{~m}^{-3}\right)\end{array}$} \\
\hline & & El Niño & Tran & La Niña & Typical & Total & SDC \\
\hline IMECOCAL 9709/10 & September-October 1997 & 103 & & & & 43 & 6 \\
\hline IMECOCAL 9801/02 & January-February 1998 & 100 & & & & 195 & 39 \\
\hline IMECOCAL 9809/10 & September-October 1998 & & 65 & & & 4 & 2 \\
\hline IMECOCAL 9901 & January 1999 & & & 46 & & 145 & \\
\hline \multirow[t]{2}{*}{ IMECOCAL 9908} & August 1999 & & & 52 & & 285 & \\
\hline & & & & & & \multicolumn{2}{|c|}{ Mean $=95$} \\
\hline TOPO 0903 & & & & & & \multicolumn{2}{|c|}{ Mean $=96$} \\
\hline \multicolumn{8}{|l|}{ Gulf of California } \\
\hline S-195 & November 2004 & & & & 82 & 208 & $105^{*}$ \\
\hline GCG 0503 & March 2005 & & & & 230 & 1,102 & 29 \\
\hline GCG 0505 & May 2005 & & & & 166 & 2,671 & 309 \\
\hline GOLCA 0511 & November 2005 & & & & 541 & 408 & 15 \\
\hline S-207 & November 2006 & & & & 103 & 208 & $133^{*}$ \\
\hline GOLCA 0701 & January 2007 & & & & 139 & 401 & \\
\hline
\end{tabular}

(b)

\section{Southwest coast}

TOPO 0403

TOPO 0903
April-May 2003

September-October 2003
162

1,531

130

381
1,455

179

\section{Gulf of California}

\begin{tabular}{llrrr} 
S-195 & November 2004 & 61 & 2,759 & 2,720 \\
CGC 0509 & September 2005 & 139 & 416 & 321 \\
S-207 & November 2006 & 99 & 48 & 35 \\
\cline { 2 - 4 } & & & Mean = 1,025 \\
\hline
\end{tabular}




\section{ACKNOWLEDGMENTS}

RMGG was granted a scholarship by the National Council of Science and Technology (CONACYT, Mexico) and the Instituto Politécnico Nacional (BEIFI program) through projects SIP 20150609 and SIP 20161524. RDS and RAI hold scholarships from the Comisión de Operación y Fomento de Actividades Académicas and Programa de Estímulos al Desempeño de los Investigadores. The Secretaría de Marina, Armada de México (Mexican Navy), provided sampling logistics through their research projects.

English translation by Claudia Michel-Villalobos.

\section{REFERENCES}

Aceves-Medina G, De Silva-Dávila R, Cruz-Estudillo I, Durazo R, Avendaño-Ibarra R. 2017. Influence of the oceanographic dynamic in size distribution of cephalopod paralarvae in the southern Mexican Pacific Ocean (rainy seasons 2007 and 2008). Lat. Am. J. Aquat. Res. 45(2): 356-369.

http://dx.doi.org/10.3856/vol45-issue2-fulltext-11

Alejo-Plata MC, García-Guillén R, Herrera-Galindo J. 2013. Paralarvas y juveniles de cefalópodos en el Pacífico sur de México = Paralarvae and juvenile of cephalopods in the Mexican South Pacific. Hidrobiológica 23(2): 250-264.

Avendaño-Ibarra R, De Silva-Dávila R, Ordóñez-Guillén FE, Vázquez-López G. 2010. Composición estacional de larvas de peces frente a la costa occidental de Baja California Sur. In: Gaxiola-Castro G, Durazo R (eds.), Dinámica del Ecosistema Pelágico frente a Baja California 1997-2007: Diez Años de Investigaciones Mexicanas de la Corriente de California. Secretaría de Medio Ambiente y Recursos Naturales, Mexico City, pp. 413-430.

Beers JR. 1976. Volumetric methods. In: Steedman HF (ed.), Zooplankton Fixation and Preservation. Monographs on Oceanographic Methodology, No. 4. UNESCO Press, Paris, pp. 56-60.

Birk MA, Paight C, Seibel BA. 2017. Observations of multiple pelagic egg masses from small-sized jumbo squid (Dosidicus gigas) in the Gulf of California. J. Nat. Hist. 51(43-44): 2569-2584. http://dx.doi.org/10.1080/00222933.2016.1209248

Bolstad KSR. 2010. Systematics of the Onychoteuthidae Gray, 1847 (Cephalopoda: Oegopsida). Zootaxa 2696: 1-186.

Boyle P, Rodhouse P. 2005. Cephalopods: Ecology and fisheries. Blackwell Publishers, Oxford, 452 pp.

Camarillo-Coop S. 2006. Variación espacio-temporal de paralarvas de calamares (Cephalopoda: Ommastrephidae) de importancia comercial en la costa occidental de la Península de Baja California. MSc thesis, Centro Interdisciplinario de Ciencias Marinas-Instituto Politécnico Nacional, La Paz (Baja California Sur, Mexico), 92 pp.

Camarillo-Coop S, Salinas-Zavala CA, Lavaniegos BE, Markaida U. 2013. Food in early life stages of Dosidicus gigas (Cephalopoda: Ommastrephidae) from the Gulf of California, Mexico. J. Mar. Biol. Assoc. U.K. 93(7): 1903-1910.

http://dx.doi.org/10.1017/S0025315413000398

Camarillo-Coop S, Salinas-Zavala CA, Manzano-Sarabia M, Aragón-Noriega EA. 2011. Presence of Dosidicus gigas paralarvae (Cephalopoda: Ommastrephidae) in the central Gulf of California, Mexico related to oceanographic conditions. J. Mar. Biol. Assoc. U.K. 91(4): 807-814.

https://doi.org/10.1017/S0025315410001517 ecuatorial tropical (Staaf et al. 2013) como en el golfo de California (De Silva-Dávila 2013) y en la CSO (este estudio), son evidencia sólida de que este tipo de arrastre de bajo costo, fácil ejecución y con menor tiempo de operación puede ser el mejor para iniciar un programa de seguimiento y evaluación del recurso calamar frente al noroeste de México. Las diferencias registradas en los muestreos con arrastres superficiales y oblicuos analizadas por separado pueden llevar a inferencias y conclusiones diferentes en el estudio de la comunidad completa de paralarvas.

\section{Agradecimientos}

RMGG recibió una beca de posgrado del Consejo Nacional de Ciencia y Tecnología (México) y del Instituto Politécnico Nacional (programa BEIFI) a través de los proyectos SIP 20150609 y SIP 20161524. RDS y RAI son becarios de la Comisión de Operación y Fomento de Actividades Académicas y del Programa de Estímulos al Desempeño de los Investigadores. La Secretaría de Marina, Armada de México, proveyó la logística para el muestreo a través de sus proyectos de investigación.

Chen DS, Dykhuizen GV, Hodge J, Gilly WF. 1996. Ontogeny of copepod predation in juvenile squid (Loligo opalescens). Biol. Bull. 190(1): 69-81.

http://dx.doi.org/10.2307/1542676

[CONAPESCA] Comisión Nacional de Acuacultura y Pesca. 2014. Anuario Estadístico de Acuacultura y Pesca de la Comisión Nacional de Acuacultura y Pesca. SAGARPA-CONAPESCA, Mazatlán (Sinaloa, Mexico), 295 pp.

De Silva-Dávila R. 2013. Paralarvas de cefalópodos en el Golfo de California, México. Dissertation, Universidad de Guadalajara, Centro Universitario de la Costa Sur, Mexico, $370 \mathrm{pp}$.

De Silva-Dávila R, Franco-Gordo C, Hochberg FG, GodínezDomínguez E, Avendaño-Ibarra R, Gómez-Gutiérrez J, Robinson CJ. 2015. Cephalopod paralarval assemblages in the Gulf of California during 2004-2007. Mar. Ecol. Prog. Ser. 520: 123-141. http://dx.doi.org/10.3354/meps11074

De Silva-Dávila R, Palomares-García R, Martínez-López A, Carballido-Carranza MA. 2002. Standing stock of Nyctiphanes simplex in the southern region of the California Current System. J. Plankton Res. 24(10): 1057-1066. http://dx.doi.org/10.1093/plankt/24.10.1057

Durazo R. 2015. Seasonality of the transitional region of the California Current System off Baja California. J. Geophys. Res. Oceans 120(2): 1173-1196. http://dx.doi.org/10.1002/2014JC010405

Durazo R, Baumgartner TR. 2002. Evolution of oceanographic conditions off Baja California: 1997-1999. Prog. Oceanogr. 54(1-4): 7-31. http://dx.doi.org/10.1016/S0079-6611(02)00041-1

Fleminger A. 1964. Distributional atlas of calanoid copepods in the California Current region, Part I. CalCOFI Atlas 2, 313 pp.

González AF, Otero J, Guerra A, Prego R, Rocha FJ, Dale AW. 2005. Distribution of common octopus and common squid paralarvae in a wind-driven upwelling area (Ria of Vigo, northwestern Spain). J. Plankton Res. 27(3): 271-277. https://dx.doi.org/10.1093/plankt/fbi001

Granados-Amores J, De Silva-Dávila R, Camarillo-Coop S, Hochberg FG, Durazo R, Avendaño-Ibarra R. 2010. 
Composición de especies y patrones de distribución de paralarvas de calamar, 1996-1999. In: Gaxiola-Castro G, Durazo R. (eds.), Dinámica del Ecosistema Pelágico frente a Baja California 1997-2007: Diez Años de Investigaciones Mexicanas de la Corriente de California. Secretaría de Medio Ambiente y Recursos Naturales, Mexico City, pp. 453-467.

Hernández-Muñoz AT, Rodríguez-Jaramillo C, Mejía-Rebollo A, Salinas-Zavala CA. 2016. Reproductive strategy in jumbo squid Dosidicus gigas (D’Orbigny, 1835): A new perspective. Fish. Res. 173(2): 145-150. http://dx.doi.org/10.1016/j.fishres.2015.09.005

Hoving HJT, Gilly WF, Markaida U, Bernoit-Bird KJ, Brown ZW, Daniel P, Field JC, Parassenti L, Liu B, Campos B. 2013. Extreme plasticity in life-history strategy allows a migratory predator (jumbo squid) to cope with changing climate. Glob. Change Biol. 19(7): 2089-2103.

https://dx.doi.org/10.1111/gcb.12198

Jereb P, Roper CFE. 2010. Cephalopods of the world. An annotated and illustrated catalogue of cephalopod species known to date. Myopsid and Oegopsid Squids. FAO Species Catalogue for Fishery Purposes, No. 4, vol. 2, Food and Agriculture Organization, Rome, $605 \mathrm{pp}$.

Jereb P, Roper CFE, Norman MD, Finn KJ. 2014. Cephalopods of the world. An annotated and illustrated catalogue of cephalopod species known to date. Octopods and Vampire Squids. FAO Species Catalogue for Fishery Purposes, no. 4, vol. 3, Food and Agriculture Organization, Rome, 370 pp.

Jiménez-Rosenberg SPA, Saldierna-Martínez RJ, Aceves-Medina G, Hinojosa-Medina A, Funes-Rodríguez R, Hernández-Rivas M, Avendaño-Ibarra R. 2010. Fish larvae off the northwestern coast of the Baja California Peninsula, Mexico. Check List 6(2): 334-349. http://dx.doi.org/10.15560/6.2.334

Kramer D, Kalin MJ, Stevens EG, Thrailkill JR, Zweifel JR. 1972. Collecting and processing data on fish eggs and larvae in the California Current region. NOAA Tech. Rep. NMFS circ. 370, USA, 38 pp.

Lavaniegos BE, Jiménez-Pérez LC, Gaxiola-Castro G. 2002. Plankton response to El Niño 1997-1998 and La Niña 1999 in the southern region of the California Current. Prog. Oceanogr. 54(1-4): 33-58. https://dx.doi.org/10.1016/S0079-6611(02)00042-3

Lea RN, Rosenblatt RH. 2000. Observation on fishes associated with the 1997-98 El Niño off California. CalCOFI Rep. 41: 117-129.

Lynn RJ, Simpson JJ. 1987. The California Current System: The seasonal variability of its physical characteristics. J. Geophys. Res.: Oceans 92(C12): 947-966. https://dx.doi.org/10.1029/JC092iC12p12947

Margalef R. 1982. Ecología. Editorial Omega, Barcelona, 951 pp.

Moreno A, Dos Santos A, Piatkowski U, Santos AMP, Cabral H. 2009. Distribution of cephalopod paralarvae in relation to the regional oceanography of the western Iberia. J. Plankton Res. 31(1): 73-91. https://dx.doi.org/10.1093/plankt/fbn103

McCune B, Mefford MJ. 2011. PC-ORD. Multivariate Analysis of Ecological Data. Version 6.0 MjM Software, Gleneden Beach, OR (USA).

Nesis KN. 2003. Distribution of Recent Cephalopoda and implications for plio-pleistocene events. In: Warnke K, Keupp H, Boletzky SV (eds.), Coleoid Cephalopods Through Time. Proceedings of an international symposium, 1st ed. Berliner Paläobiol. Abh. Berlin, pp. 199-224.

O’Dor RK, Foy EA, Helm PL, Balch N. 1986. The locomotion and energetics of hatchling squid, Ilex illecebrosus. Am. Malacol. Bull. 4: 55-60.

Piatkowski U, Pierce GJ, Morais da Cunha M. 2001. Impact of cephalopods in the food chain and their interaction with the environment and fisheries: an overview. Fish. Res. 52(1-2): 5-10. https://dx.doi.org/10.1016/S0165-7836(01)00226-0

Roper CFE, Voss GL. 1983. Guidelines for taxonomic descriptions of cephalopod species. Mem. Natl. Mus. Melbourne (Victoria) 44:48-63. https://doi.org/10.24199/j.mmv.1983.44.03

Saito K. 1994. A sampling method for rynchoteuthion paralarvae of neon flying squid Ommastrephes bartramii. Bull. Hokkaido Nat. Fisheries Res. Inst. 58: 25-34.

Shea EK. 2005. Ontogeny of the fused tentacles in three species of ommastrephid squids (Cephalopoda, Ommastrephidae). Invertebr. Biol. 124(1): 25-38. https://doi.org/10.1111/j.1744-7410.2005.1241-04.x

Staaf DJ, Camarillo-Coop S, Haddock SHD, Nyack AC, Payne J, Salinas-Zavala CA, Seibel BA, Trueblood L, Widmer C, Gilly WF. 2008. Natural egg mass deposition by the Humboldt squid (Dosidicus gigas) in the Gulf of California and characteristics of hatchlings and paralarvae. J. Mar. Biol. Assoc. U.K. 88(04): 759-770. https://doi.org/10.1017/s0025315408001422

Staaf DJ, Redfern JV, Gilly WF, Watson W, Ballance LT. 2013. Distribution of ommastrephidae paralarvae in the eastern tropical Pacific. Fish. Bull. 111(1): 78-89.

https://dx.doi.org/10.7755/FB.111.1.7

Staaf DJ, Zeidberg LD, Gilly WF. 2011. Effects of temperature on embryonic development of the Humboldt squid Dosidicus gigas. Mar. Ecol. Prog. Ser. 441: 165-175. https://doi.org/10.3354/meps09389

Sweeney MJ, Roper CFE, Mangold KM, Clarke MR, Boletzky SV. 1992. "Larval" and juvenile cephalopods: A manual for their identification. Smith. Contrib. Zool. 513: 1-282.

https://dx.doi.org/10.5479/si.00810282.513

Ter Braak CJF. 1994. Canonical community ordination. Part I: Basic theory and linear methods. Écoscience 1(2): 127-140. http://dx.doi.org/10.1080/11956860.1994.11682237

Vidal EAG, Haimovici M. 1998. Feeding and the possible role of the proboscis and mucus cover in the ingestion of the microorganisms by rhynchoteuthion paralarvae (Cephalopoda: Ommastrephidae). Bull. Mar. Sci. 63(2): 305-316.

Vidal EAG, Haimovici M, Hackbart VCS. 2010. Distribution of paralarvae and small juvenile cephalopods in relation to primary production in an upwelling area off southern Brazil. ICES J. Mar. Sci. 67(7): 1346-1352. https://dx.doi.org/10.1093/icesjms/fsq080

Young RE, Harman RF. 1988. "Larva", "paralarvae" and "subadult" in cephalopod terminology. Malacologia 29(1): 201-207.

Zaitsev O, Trasviña-Castro A, Linero-Cueto J, Gaxiola-Castro G, Cepeda-Morales J. 2014. Oceanographic conditions over the continental shelf off Magdalena Bay (Mexico) in 2011-2012 = Condiciones oceanográficas en la plataforma continental frente a bahía Magdalena (México) en 2011-2012. Cienc. Mar. 40(2): 89-112. http://dx.doi.org/10.7773/cm.v40i2.2314

Zaragoza N, Quetglas A, Hidalgo M, Álvarez-Berastegui D, Balbín R, Alemany F. 2014. Effects of contrasting oceanographic conditions on the spatiotemporal distribution of Mediterranean cephalopod paralarvae. Hydrobiologia 749(1): 1-14. http://dx.doi.org/10.1007/s10750-014-2132-x

Zepeda-Benitez VY, Morales-Bojórquez E, Díaz-Uribe JG, Nevárez-Martínez MO, Hernández-Herrera A, López-Martínez J. 2017. Implementation of catch-at-age model for the jumbo squid Dosidicus gigas. Ecol. Modell. 344: 6-16. https://doi.org/10.1016/j.ecolmodel.2016.10.019

Received March 2017, accepted February 2018. 